\title{
Aerodynamics of Pitching Wings: Theory and Experiments
}

\author{
Huai-Te $\mathrm{Yu}^{1}$ and Luis P. Bernal ${ }^{2}$ \\ University of Michigan, Ann Arbor, MI, 48109 \\ Kenneth Granlund ${ }^{3}$ and Michael V. Ol ${ }^{4}$ \\ Air Force Research Lab, Wright-Patterson AFB
}

\begin{abstract}
The unsteady aerodynamics of pitching wings at high reduced pitch rate is investigated experimentally and theoretically. Simple potential flow analysis is used to compute lift, drag and pitching moment, and compared to experimental measurements. The wing motion is a linear pitch ramp between 0 and 45 degrees with smoothing at the start and end of the motion. Recent experimental results are reported for several reduced pitch rates in the range $K=0.06$ and 0.39 which corresponds to pitch times of 1 and 6 convective times, respectively, and for several wing planform geometries, pivot locations and Reynolds numbers. It is shown that the lift during the motion is in agreement with linear potential flow theory including rotation rate and finite span effects. The theoretical predictions significantly underestimates drag coefficients in the measurement. At high rotation rates the wing planform shape significantly impacts aerodynamic force for leading edge pivot with a triangular wing producing $25 \%$ more transient lift than trapezoidal and rectangular wings. The effect of Reynolds number and smoothing kinematics are investigated experimentally. At high reduced pitch rates a longer smoothing transient produces larger transient lift coefficients.
\end{abstract}

\section{Nomenclature}

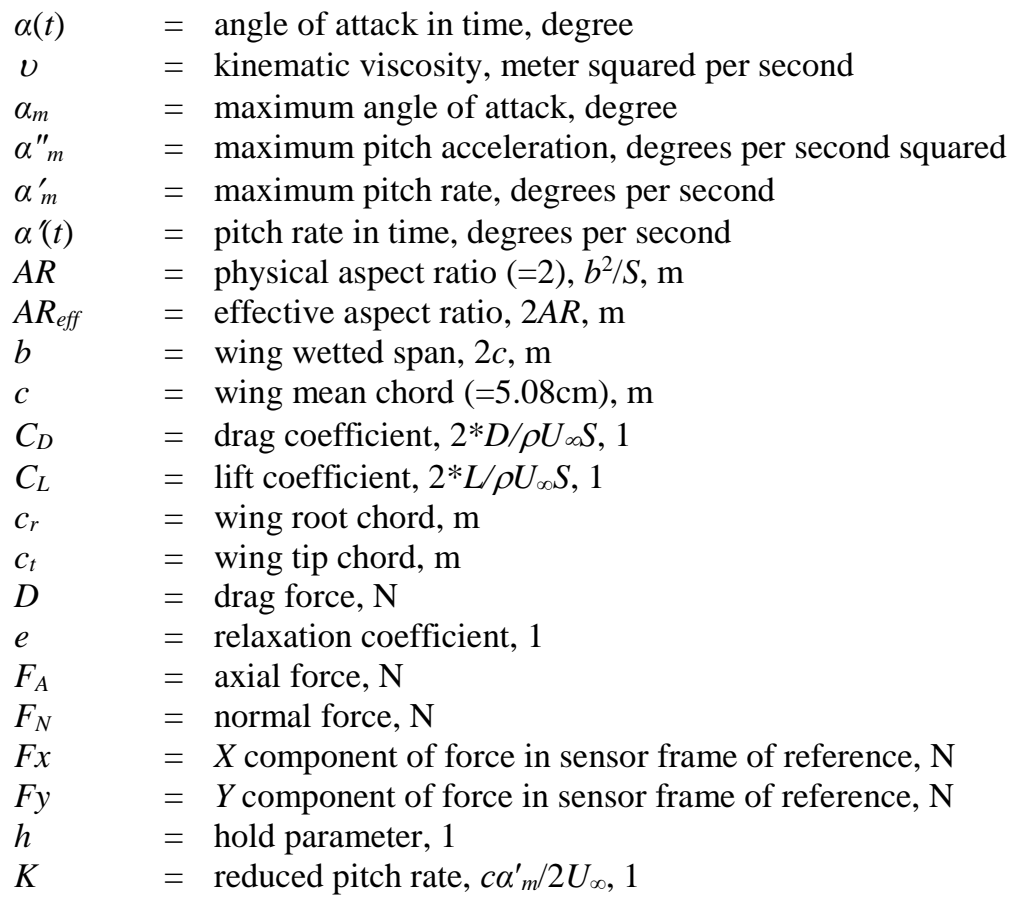

\footnotetext{
${ }^{1}$ Graduate Research Assistant, Department of Aerospace Engineering, University of Michigan, htyu@ @umich.edu.

${ }^{2}$ Professor, Department of Aerospace Engineering, University of Michigan, 1pb@umich.edu.

${ }^{3}$ Air Vehicles Directorate, Wright-Patterson AFB, Kenneth.granlund@ wpafb.af.mil.

${ }^{4}$ Air Vehicles Directorate, AFRL/VAAA, Michael.Ol@ wpafb.af.mil.
}

American Institute of Aeronautics and Astronautics 
$=$ point in time when a wing starts to pitch-up in an unsmooth motion trace, second

$=$ point in time when a wing starts to hold in an unsmooth motion trace, $\mathrm{s}$

$=$ point in time when a wing returns back to initial position in an unsmooth motion trace, $\mathrm{s}$

$=$ point in time when a wing starts to return in an unsmooth motion trace, $\mathrm{s}$

$=$ convective time, $c / U_{\infty}, \mathrm{s}$

$=$ pitch time, $\alpha_{m} / \alpha_{m}^{\prime}, \mathrm{s}$

$=$ free-stream velocity, $\mathrm{m} / \mathrm{s}$

$=$ taper ratio, $c_{t} / c_{r}, 1$

\section{Introduction}

$\mathrm{N}$ atural flyers demonstrate remarkable flight agility and efficiency. Birds and insects are able to generate relatively large forces very quickly in response to gust and other wind disturbances as well as during perching maneuvers. These flight features are of considerable interest to biologists and engineers, but remain poorly understood. They have been the subject of numerous research efforts and motivate the present paper. The transient aerodynamic force and flow development during the pitching motion of a wing is a canonical flow problem encountered in flapping wings and perching maneuvers of fixed wing vehicles. These phenomena are also relevant to the development of advanced small Micro Air Vehicles which might take advantage of the large forces generated at high pitch rates. The common features of the flows by pitching wings are leading edge vortices (LEV) and wingtip vortices (TV). The main goal of the present research is to determine i) the interplay between time scales of the wing motion and flow convection, and ii) its impact on force development and flow development for different wing geometries and pivot axis locations.

The focus of the present research is to experimentally determine the unsteady flow field about pitching finiteaspect-ratio flat-plate wings. The wing kinematics is similar to a linear pitch ramp, hold and return wing motion proposed by the AIAA Fluid Dynamics Technical Committee (FDTC) Low Reynolds Number Discussion Group $(\mathrm{LRDG})^{1}$. This motion kinematics is relevant to the study of the aerodynamics of aggressive perching maneuvers; maneuvering angle of attack is well beyond quasi-steady stall angle to produce large lift and drag forces. Ol et al. ${ }^{1}$ provide an overview of the main features of the flow dynamics. Large LEV forms during the pitch-up part of the motion and detach as the flow evolves in time. It is found that moderate values of the reduced pitch rate modify the flow significantly and cause an increase in lift well above the quasi-steady value for leading-edge pivot axis.

In a relevant study Granlund $e t a l .^{2}$ consider the aerodynamics of a pitch-up maneuver and extend prior results to a maximum pitch angle of 90 degrees. They found that both pivot location and reduced frequency play important roles on aerodynamic loads. Pitching about the leading edge produces significantly higher lift in the initial acceleration part of the motion even at low reduced pitch rates. Similar motion kinematics was investigated by Yeon et al. ${ }^{3}$; they derived the aerodynamic forces from the PIV data instead of direct measurement. The PIV data was also used to determine the LEV and TEV vortex evolution. At moderate reduced pitch rate $(K=0.2)$ a strong LEV vortex forms early in the motion followed by a relatively weak TEV. However, at high reduced pitch rate $(K=1)$ the TEV forms before the LEV.

Ol et al. ${ }^{4}$ also consider the pitch return part of the motion and found that during pitch up the lift coefficient normalized by reduced pitch rate is a simple function of the rate of change of the angle of attack. During pitch down the flow exhibits history effects from preceding parts of the motion, which invalidate the simple reduced pitch rate scaling. Other contributions using computational techniques have been reported by Eldredge et al. ${ }^{5}$, Garmann and $\mathrm{Visbal}^{6}$, and Lian and $\mathrm{Ol}^{7}$.

Harper and Flanigan ${ }^{8}$ consider the effect of pitch rate on the maximum lift coefficient of a model aircraft and show an increase in maximum lift coefficient with pitch rate along the steady lift-vs.-angle-of-attack curve. Work on dynamic stall has documented that the increase in lift is associated with the formation of a leading edge vortex (LEV) ${ }^{9}$. Currier and Fung ${ }^{10}$ investigated the onset of dynamic stall ( $K$ in the range from 0 to 0.19 ), and suggested that the benefit from dynamic stall is present only during the separation process and inviscid theory could be used to predict the flow before stall. Additionally, leading edge vortices have been shown by Ellington et al. ${ }^{11}$ to be important for insect flight. They visualized the flow about a hovering hawkmoth model and showed the formation of leading edge and tip vortices. A spiral LEV was observed along the span of the venation-like wing. They concluded that this LEV is created by the translation motion not by wing rotation. The spiral vortex, however, was not found by Birch and 


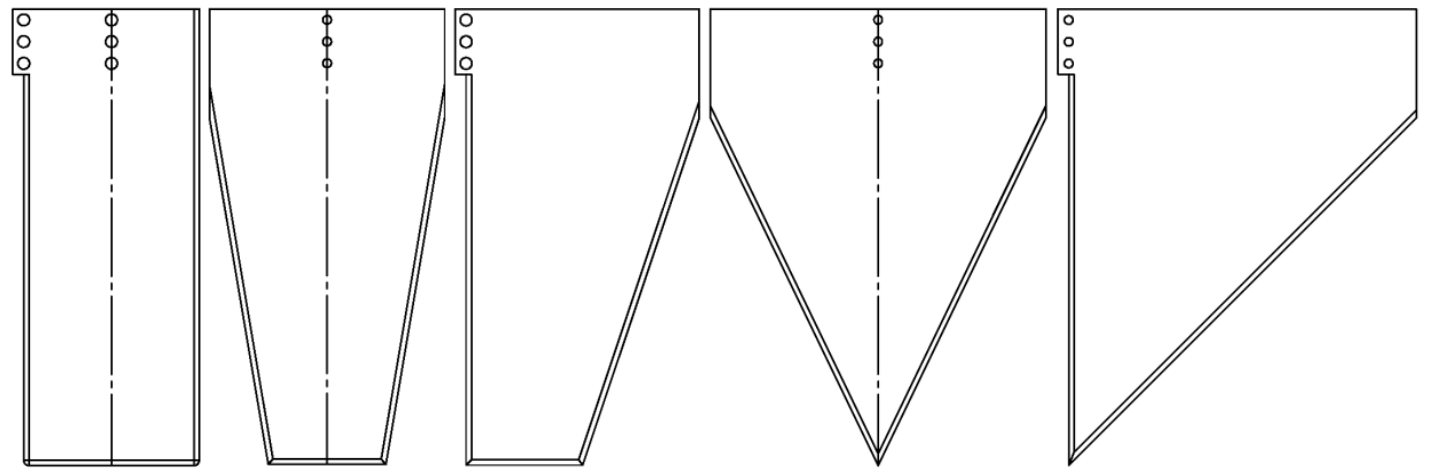

Figure 1 Illustration of different planform wings: (from left to right) rectangular wing, two trapezoidal wings (isosceles and right), two triangular wings (isosceles and right).

Dickson $^{12}$ in an investigation of LEV attachment of a hovering fruit fly model, they suggested the downwash induced by the tip vortex and wake vorticity may stabilize the LEV and maintain the LEV attached during the flapping motion. Computational results of hawkmoth and fruit fly in hover by Shyy and Liu ${ }^{13}$ showed that higher Reynolds number generates more pronounced axial flow; the LEV is a helical structure and breaks down during the flapping motion. At lower Reynolds number the weaker axial flow stabilizes the LEV which is attached during the entire flapping stroke, although it breaks down during wing supination. In the present work we examine the development of aerodynamic forces and its relation to vortex development over the wing, which is relevant to biological flyers including very rapid pitch-up motions relevant to aggressive maneuvers of small flyers.

In the present paper we consider flat plate wings and focus on wing planform effects and simple theoretical analyses that can provide valuable predicting capability. Experiments are conducted at Reynolds numbers in the range of 5,000 and 14,000, and reduced pitch rate of 0.06 and 0.39 . We consider rectangular, trapezoidal and triangular wings and pivot-axis locations at leading edge, mid-chord and trailing edge.

\section{Experimental Setup}

\section{A. Wing Configuration and Water Tunnel}

The experiments were conducted in the low-turbulence water tunnel at University of Michigan, which has cross section $61 \mathrm{~cm}$ wide and $61 \mathrm{~cm}$ height. This tunnel produces steady free stream velocity from $5 \mathrm{~cm} / \mathrm{s}$ to $40 \mathrm{~cm} / \mathrm{s}$.

Finite-aspect-ratio wings with effective aspect ratio 4 were used in the present study. All wings have the same mean chord length, which is the wing area divided by wing span. Wing planform geometries include: a rectangular wing, two trapezoidal wings (isosceles and right), and two triangular wings (isosceles and right), as shown in Fig. 1. The mounting holes near the top edge of the wings are used to attach the wing to a sensor adapter, and then attached to a force transducer. These mounting holes are aligned with the pitch axis and also coincident with the axis of the force transducer. The force transducer measures forces in three directions: the chord direction (x-axis), the direction normal to the wing surface (y-axis), and spanwise direction (z-axis). At the same time, the three corresponding torques are also recorded.

Three pivot axes are considered, including leading edge (LE) pivot, mid-chord (MC) pivot, and trailing edge (TE) pivot. The right trapezoidal and triangular wings are used for tests at both LE and TE pivot axes; the isosceles wings are for tests at MC pivot axis. The TE pivot wing configuration was conducted by simple rotating the wing

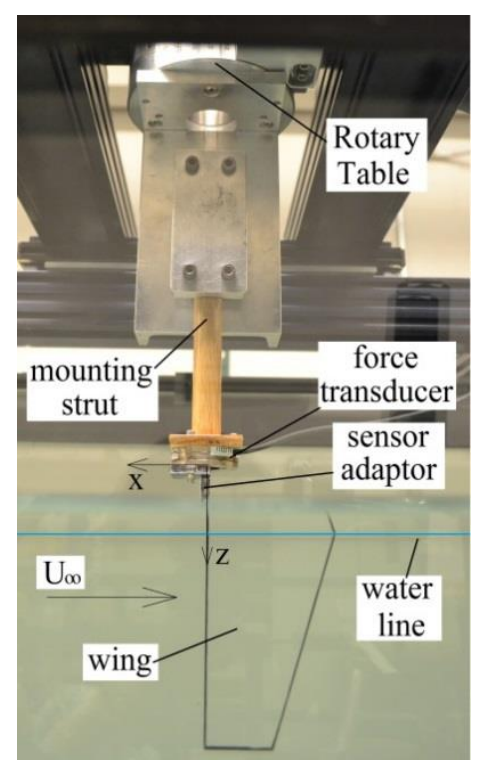

Figure 2 Wing configuration for force measurement. at LE pivot axis by $180^{\circ}$ to position the pivot axis at downstream, which results in sign change of measured forces (Fx and $\mathrm{Fy}$ ) and torques (Tx and Ty) in sensor frame of reference. For MC pivot axis, the wing configuration has the same sensor frame of reference as wing configuration at LE pivot axis, but uses the wing with mounting holes at mid-chord.

American Institute of Aeronautics and Astronautics 


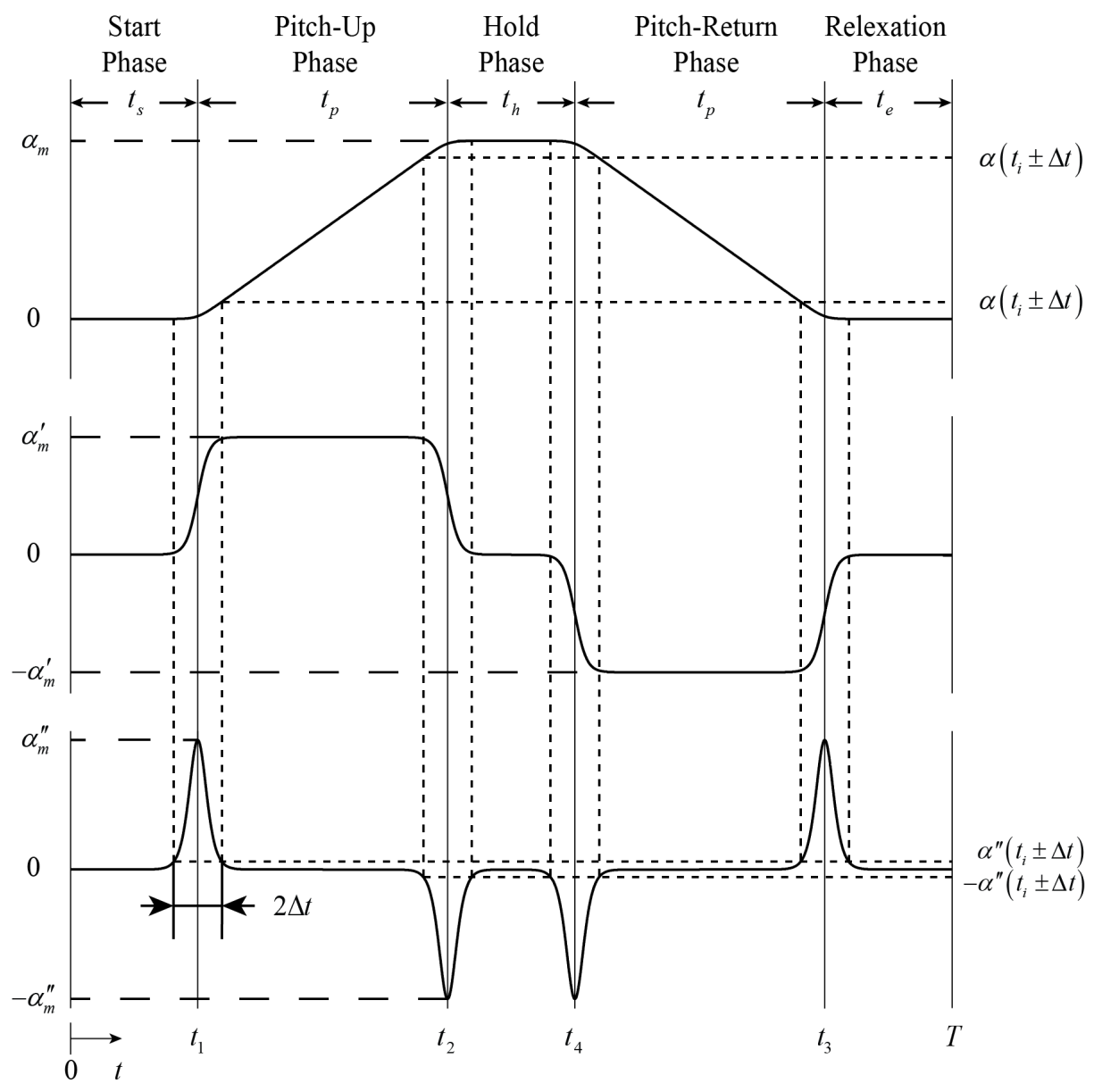

Figure 3 Illustration of linear pitch-hold-return kinematics.

A picture of force measurement setup is shown in Fig. 2, which illustrates the trapezoidal wing at LE pivot axis. The wing model is partially submerged in the water tunnel with the free surface providing a plane of symmetry for the flow.

\section{B. Wing Kinematics}

The wing motion is a linear pitch ramp from $0^{\circ}$ to $45^{\circ}$ with smoothing at the beginning and the end of the pitch ramp. The wing motion is divided into five phases: start phase, pitch-up phase, hold phase, pitch-return phase, and relaxation phase. The time duration for each phase is defined in Fig. 4.

The implementation of wing kinematics uses a smoothing function to minimize model vibration at transition corners, which are denoted by $t_{1}$ through $t_{4}$ as shown in Fig. 4. This smoothing function is given in Eq. (1), a modified form of the smoothing function used by Eldredge et al (2009). The first derivative and the second derivative of the function give the pitch rate and pitch acceleration, respectively. The temporal duration, pitch angle change, and maximum acceleration during the smoothing regions are controlled by parameter $B$, yielding discernible regions of constant pitch rate and pitch acceleration.

$$
\alpha(t)=\frac{\alpha_{m}}{2 B} \sum_{i=1}^{4}(-1)^{i+1} \ln \left\{\cosh \left[B\left(t-t_{i}\right) / t_{p}\right]\right\}, i=1,2,3,4
$$

where

$$
B=2 t_{p} / t_{a}=\cosh ^{-1}(\sqrt{1 / \gamma}) / \beta
$$

The parameter $B$ is the ratio of pitch time $\left(t_{p}=\alpha_{m} / \alpha_{m}^{\prime}\right)$ to pitch acceleration time $\left(t_{a}=\alpha_{m}^{\prime} / \alpha_{m}^{\prime \prime}\right)$, and is analogous to $a \alpha_{m} / 2 K$ in Eldredge et al.'s original formulation ( $a$ is free parameter and $K$ is the reduced pitch rate) and $A \alpha_{m}$ in $Y u$ and Bernal (2013). This parameter $B$ can also be obtained by specifying $\beta$ and $\gamma$. The $\beta$ is a ratio of smoothing angle to the maximum angle of attack, $\Delta \alpha / \alpha_{m}$. The $\gamma$ is a ratio of margin acceleration to the maximum acceleration,

American Institute of Aeronautics and Astronautics 


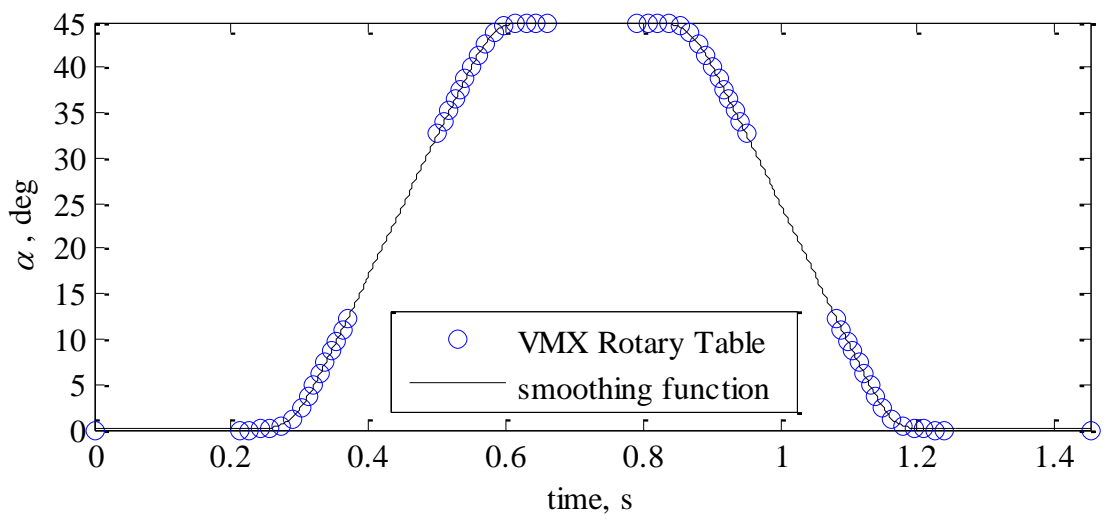

Figure 4 An illustration of wing kinematic implementation.

$\left|\alpha{ }^{\prime \prime}\left(t_{i}+\Delta t\right)\right| / \alpha_{m}{ }_{m}$ during the smoothing. The duration in the pitch-up phase is the same as the duration of the pitch-return phase; other phase durations are defined in Eqs. (2)-(4).

$$
\begin{aligned}
t_{s} & =s t_{c} \\
t_{h} & =h t_{c} \\
t_{e} & =e t_{c}
\end{aligned}
$$

In terms of convective times, the start duration denoted by $s$ is one convective time $(s=1)$. The hold duration is at least 130 convective times $(h>130)$ to ensure the flow reaching steady conditions at the maximum pitch angle of $45^{\circ}$. The relaxation duration is about 30 convective times $(e>30)$ to return the flow to the initial undisturbed condition.

Wing motions are implemented using a Velmex Rotary Table in discretized profile as illustrated in Fig. 5. There are 13 points in each smoothing transient. For all force measurements the wing motion is repeated 60 times and phase averages are used to obtain mean values with relatively small uncertainty. A summary of the wing kinematics parameters is provided in Table.

\section{Force Measurements}

The force sensor is a Nano 43 Force/Torque sensor manufactured by ATI Industrial Automation. The sensor's maximum calibrated load is $18 \mathrm{~N}$ and the resolution is $1 / 256 \mathrm{~N}$, in all three axes. The force sensor is attached to a Velmex rotary table driven by a stepper motor. Two rotary table were considered, depending on the requirement of the pitch rate, B4818TS has a resolution of $20 \mathrm{deg} / \mathrm{s}$ and maximum pitch rate of $200 \mathrm{deg} / \mathrm{s}$, and B4836TS has a resolution of $40 \mathrm{deg} / \mathrm{s}$ and maximum pitch rate of $100 \mathrm{deg} / \mathrm{s}$. Both the force sensor and the rotary table are located above the water surface. All wings are flat plates with mean-chord length of 2" and 2 mean-chords immersed in water. The thickness is 0.125 " (6.25\% of chord) and all edges are rounded. They are made of plexiglass sheet, mounted vertically at the center of the water channel. The wings are attached to the tool side of the sensor with an aluminum adapter designed to minimize the mass of the system, which is not more than 46.2 grams including the wing, the sensor adapter and the mounting screws. Because of the small mass, inertia forces and static weight of the model are very

\begin{tabular}{|c|c|c|c|c|c|c|c|c|c|c|c|c|}
\hline \multirow[t]{2}{*}{$\alpha_{m}^{\prime}$} & \multirow[t]{2}{*}{$\beta, \%$} & \multirow[t]{2}{*}{$\gamma, \%$} & \multirow[t]{2}{*}{$\alpha_{m}$} & \multirow[t]{2}{*}{$c$} & \multirow[t]{2}{*}{$t_{p}$} & \multirow[t]{2}{*}{$2 t_{a}$} & \multirow[t]{2}{*}{$\alpha_{m}^{\prime \prime}$} & \multirow[t]{2}{*}{$B$} & \multirow{2}{*}{$\begin{array}{c}\text { Yu \& Bernal } \\
(2013)\end{array}$} & \multicolumn{3}{|c|}{ Eldredge et al. (2009) } \\
\hline & & & & & & & & & & $a$ & $U_{\infty}$ & $K$ \\
\hline 155 & 27.2 & 1 & \multirow{4}{*}{45} & \multirow{4}{*}{2} & 0.290 & 0.105 & 2937 & 11 & 14.00 & 11 & 17.500 & $\pi / 8$ \\
\hline 76.4 & 13.9 & 1 & & & 0.588 & 0.109 & 1402 & 21.60 & 27.50 & 11 & 16.944 & 0.2 \\
\hline 37.5 & 13.9 & 1 & & & 1.199 & 0.222 & 338 & 21.60 & 27.50 & 11 & 8.314 & 0.2 \\
\hline 25.6 & 6.49 & 1 & & & 1.754 & 0.152 & 338 & 46.15 & 58.76 & 11 & 12.153 & $\overline{0.0936}$ \\
\hline
\end{tabular}
small and static calibration is unnecessary. The forces measured by the sensor (Fx and Fy) are first converted to axial

Table Wing kinematics of interest and its parameters

American Institute of Aeronautics and Astronautics 
and normal forces, $F_{A}$ and $F_{N}$, and then to components in the laboratory frame of reference using Eqs. (5)-(8) for lift force $(L)$ and drag force $(D)$ and their corresponding force coefficients.

$$
\begin{gathered}
L=-F_{A} \sin \alpha+F_{N} \cos \alpha \\
D=F_{A} \cos \alpha+F_{N} \sin \alpha \\
C_{L}=L /\left(\rho U_{\infty}^{2} S / 2\right) \\
C_{D}=D /\left(\rho U_{\infty}^{2} S / 2\right)
\end{gathered}
$$

\section{Results and Discussion}

\section{A. Potential Flow Theory}

It is found that a surprisingly useful theoretical framework for predicting aerodynamic forces is small-disturbance potential flow theory, including pitch rate and finite-aspect-ratio effects. Within this framework non-circulatory and circulatory effects can be superposed, which facilitates the analysis. Also finite span effects and wake vorticity can be incorporated using lifting line theory. In this section we review steady potential flow theory of pitching wings. It is assumed that the flow is quasi-steady and inertia effects can be ignored. Linearized theory assuming small disturbances, large aspect-ratio wings and a flat wake ${ }^{14}$ (Glauert, 1947) is used to estimate pitch rate, wing planform geometry and pivot location effects in force development ${ }^{15}$ (Leishman, 2006). Within this framework the wing aerodynamic forces are derived from the wing section properties using lifting line theory.

\section{Sectional airfoil}

The normal velocity on an airfoil is given in Eq. (9) at any instant time.

$$
U_{\infty, n}(x, t)+U_{\Omega, n}(x, t)+w^{\prime}(x, t)=0
$$

The first term is the normal velocity produced by the free-stream, the second term is from the pitching motion, and the third term is due to the bound vorticity. These are given in Eqs. (10)-(12), respectively, assuming small camberline slope $(d z / d x)$ and $z(x) / x<1$.

$$
\begin{gathered}
U_{\infty, n}(x, t)=U_{\infty}(\alpha(t)-d z / d x) \\
U_{\Omega, n}(x, t)=\alpha^{\prime}(t)\left(x-x_{p}\right) \\
w^{\prime}(x, t)=\frac{1}{2 \pi} \int_{0}^{\pi} \frac{\gamma(\theta, t) \sin \theta}{\cos \theta-\cos \theta_{x}} d \theta
\end{gathered}
$$

where $x$ indicates any point along the chord line, $x=c\left[1-\cos \left(\theta_{x}\right)\right] / 2,0 \leq \theta_{x} \leq \pi$.

Introducing the solution for the strength of a vortex sheet, as given in Eq. (13), the effective camberline, sectional lift coefficient and sectional pitching moment are obtained and given in Eqs. (14)-(16) at given angle of attack, sequentially. Also non-circulatory effects and normal velocity from vorticity in the wake are not included, as well as the flow moving in spanwise direction.

$$
\begin{gathered}
\gamma(\theta)=2 U_{\infty}\left[A_{0} \frac{1+\cos \theta}{\sin \theta}+\sum_{n=1}^{\infty} A_{n} \sin n \theta\right] \\
(d z / d x)_{\text {eff }}=d z / d x+\alpha_{m}^{\prime}\left(x_{p}-x\right) / U_{\infty}=\alpha-A_{0}+\sum_{n=1}^{\infty} A_{n} \cos n \theta_{0} \\
c_{l}=\frac{2 \rho U_{\infty} \Gamma}{\rho U_{\infty}^{2} S}=\pi\left[2 A_{0}+A_{1}\right] \\
c_{m, L E}=-\frac{\pi}{2}\left[A_{0}+A_{1}-\frac{A_{2}}{2}\right]
\end{gathered}
$$

The coefficient $A_{0}$ and $A_{n}$ are found using Eq. (17) and Eq. (18), respectively.

$$
\begin{gathered}
A_{0}=\alpha-\frac{1}{\pi} \int_{0}^{\pi}(d z / d x) d \theta_{0}+\frac{\alpha_{m}^{\prime} c}{2 U_{\infty}} \cos \theta_{p} \\
A_{n}=\frac{2}{\pi} \int_{0}^{\pi}(d z / d x) \cos n \theta_{0} d \theta_{0}+\frac{\alpha_{m}^{\prime} c}{2 U_{\infty}}
\end{gathered}
$$

American Institute of Aeronautics and Astronautics 

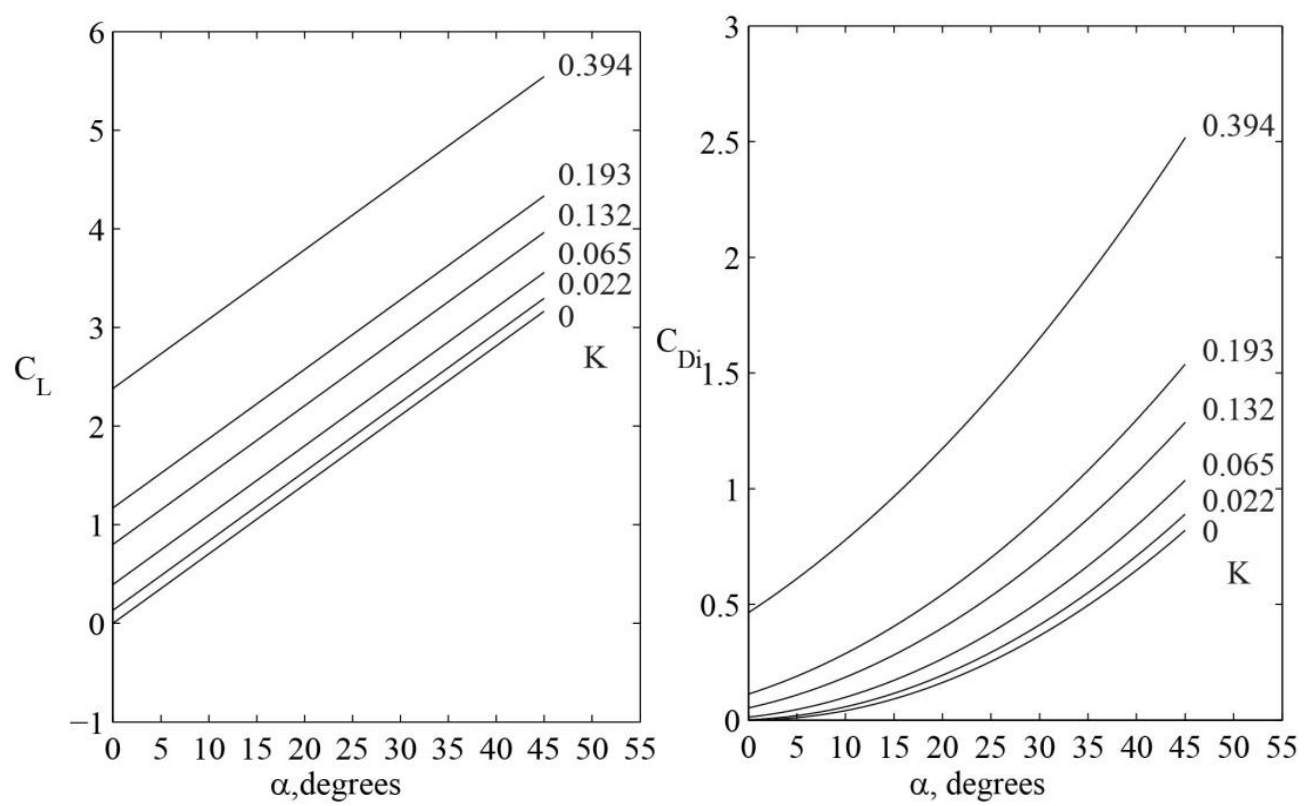

Figure 5 Force coefficients as functions of angle of attack using lifting-line theory for rectangular wing at leading edge pivot: (left) lift coefficient (right) induced drag coefficient.

where $\theta_{p}$ corresponds to the pivot axis location $x_{p}$ with $x_{p}=c\left[1-\cos \left(\theta_{p}\right)\right] / 2$.

For the present study the wings are flat plates, the corresponding sectional lift and pitching moment coefficients are given in Eq. (19) and Eq. (20), respectively.

$$
\begin{gathered}
c_{l}=a_{0}\left(\alpha-\alpha_{L=0}\right)=2 \pi\left[\alpha+K\left(\cos \theta_{p}+\frac{1}{2}\right)\right] \\
c_{m, L E}=-\frac{\pi}{2}\left[\alpha+K\left(\cos \theta_{p}+1\right)\right]
\end{gathered}
$$
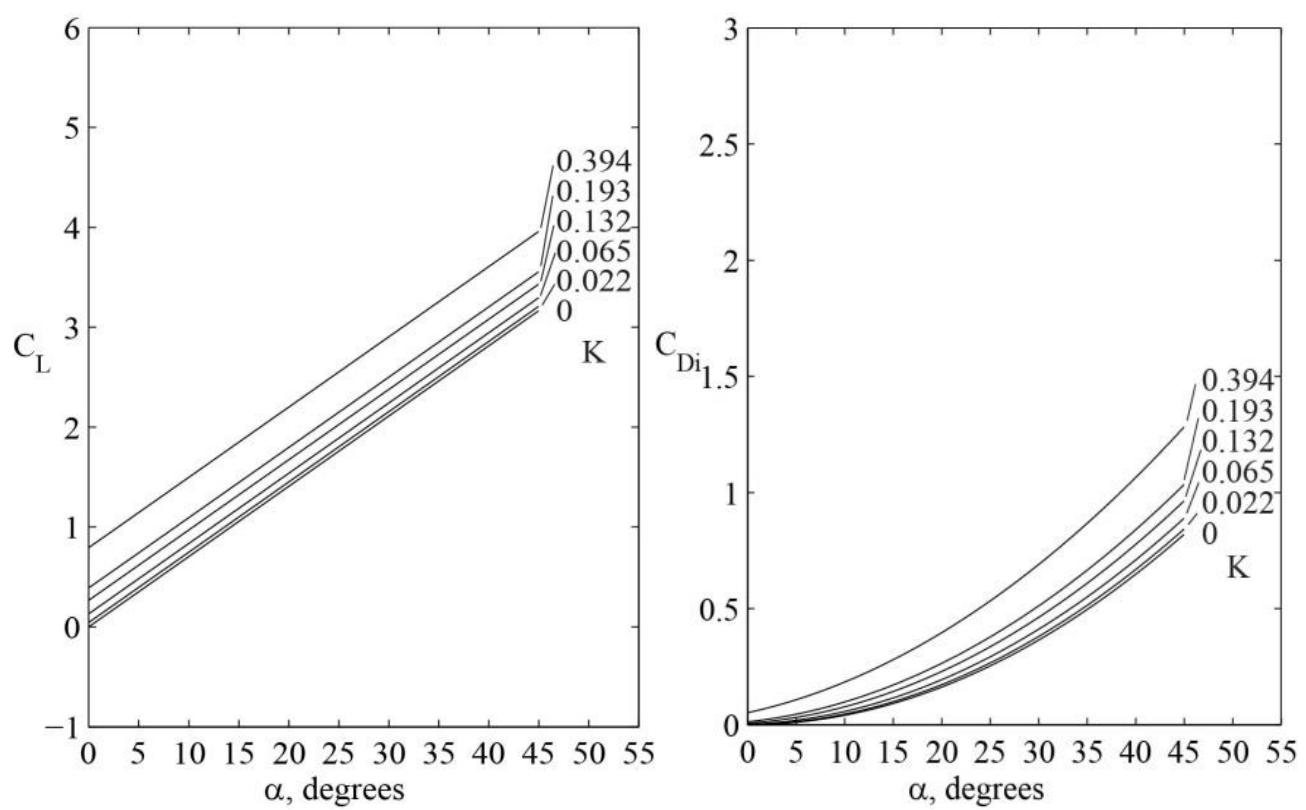

Figure 6 Force coefficients as function of angle of attack using lifting-line theory for rectangular wing at mid-chord pivot: (left) lift coefficient (right) induced drag coefficient.

American Institute of Aeronautics and Astronautics 

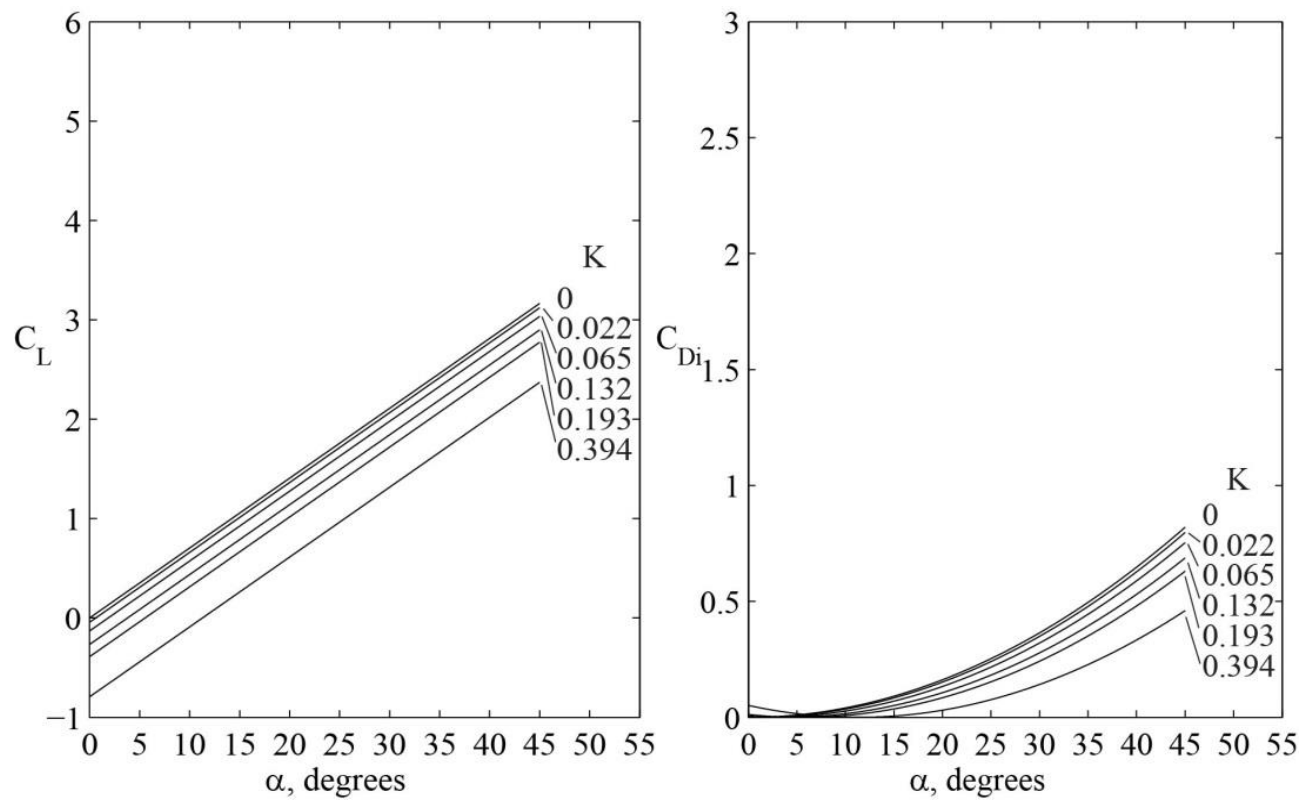

Figure 7 Force coefficients as function of angle of attack using lifting-line theory for rectangular wing at trailing-edge pivot: (left) lift coefficient (right) induced drag coefficient.

2. Finite Aspect Ratio Wing

Now considering a finite aspect-ratio wing, the lift and drag coefficients are found by introducing the slope of lift coefficient curve $\left(a_{0}\right)$ and the zero lift angle of attack $\left(\alpha_{L=0}\right)$ from sectional airfoil theory, as shown in Eq. (19). The angle of attack, including tip vortex effects, is given by Eq. (21) to determine the coefficients $D_{n}$. The first term on the right-hand side represents the induced angle of attack, and the last two terms represent the effective angle of attack.

$$
\alpha(\phi)=\sum_{n=1}^{N} n D_{n} \frac{\sin n \phi}{\sin \phi}+\frac{4 b}{a_{0} c(\theta)} \sum_{n=1}^{N} D_{n} \sin n \phi-K\left(\cos \theta_{p}+\frac{1}{2}\right)
$$

Hence, the lift and drag coefficients are then given by Eq. (22) and (23), respectively, and are shown as green curves in the following figures to represent the theoretical prediction for a rectangular wing.
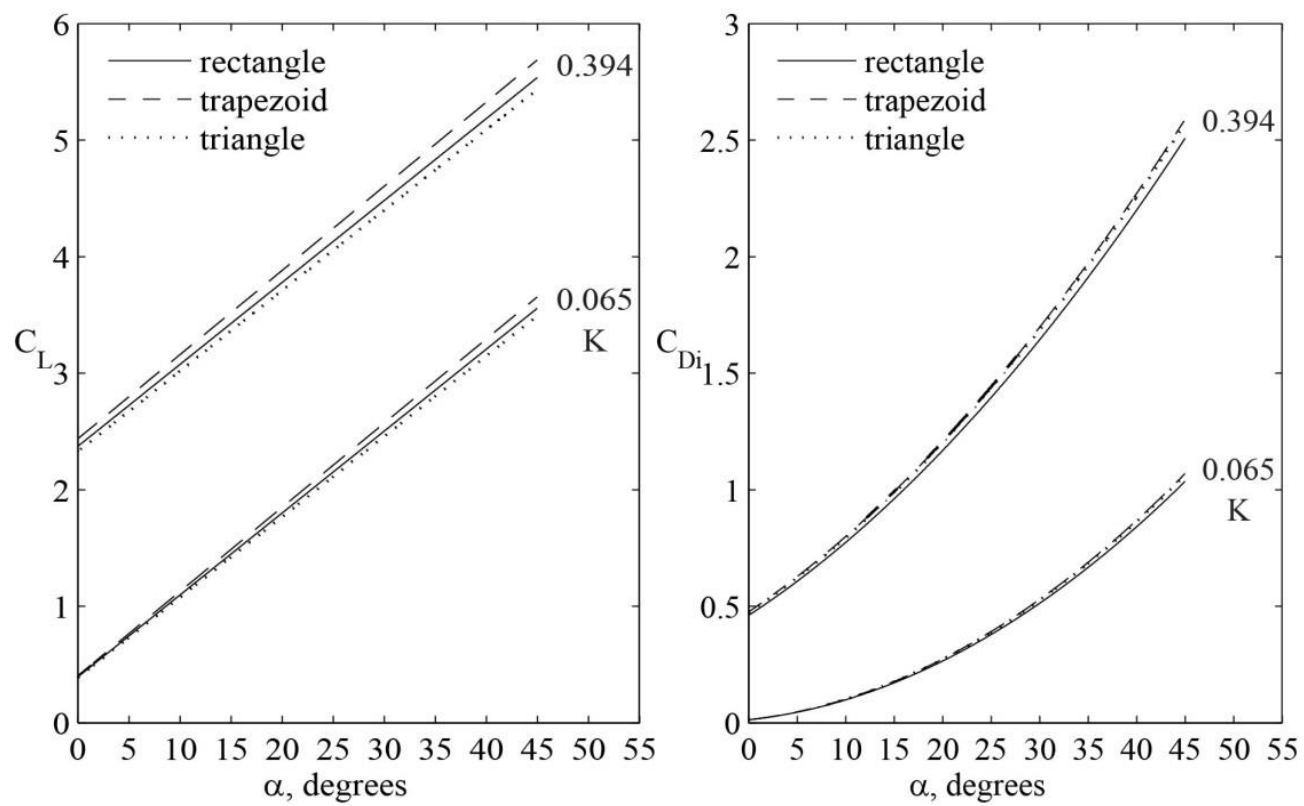

Figure 8 Force coefficients as function of angle of attack using lifting-line theory for selected wing planforms at leading edge pivot: (left) lift coefficient (right) induced drag coefficient.

American Institute of Aeronautics and Astronautics 


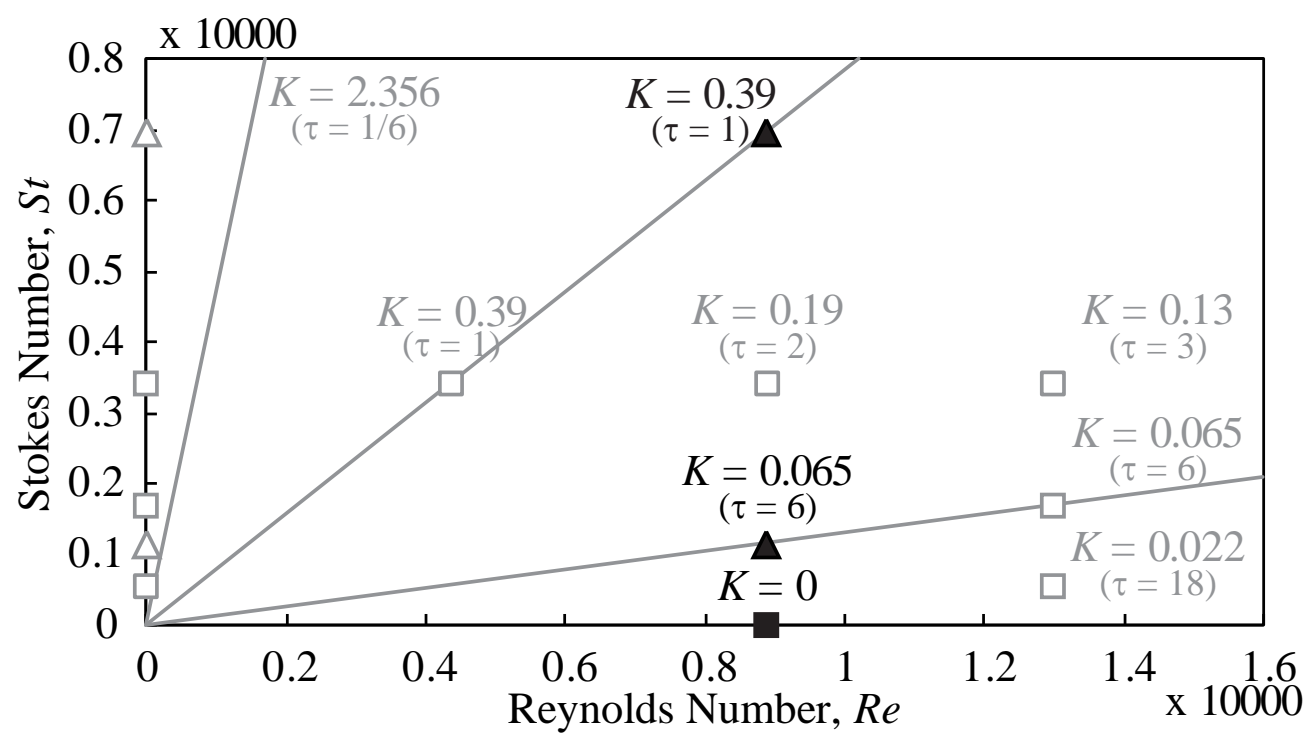

Figure 9. Experimental conditions for the study of pitch rate and wing planform effects.

$$
\begin{gathered}
C_{L}=\frac{2}{\rho U_{\infty}^{2} S} \int_{-b / 2}^{b / 2} \rho U_{\infty} \Gamma(y) d y=\frac{b^{2}}{S} \pi D_{1} \\
C_{D_{i}}=\frac{2}{\rho U_{\infty}^{2} S} \int_{-b / 2}^{b / 2} \rho U_{\infty} \Gamma(y) \alpha_{i}(y) d y=\frac{b^{2}}{S} \pi \sum_{n=1}^{N} n D_{n}^{2}
\end{gathered}
$$

Figures 6-8 show the force coefficients as a function of angle of attack for the LE, MC, and TE pivot axis, predicted using the lifting line theory presented above and $\mathrm{N}=203$ to determine $D_{n}$ coefficients. From Eqs. (19) and (20), we found that the reduced pitch rate $(K)$ effect is absent when pivot axis is at three-quarter chord. The force coefficients as shown in Figs. 6-7, where the pivot axis locations are before the three-quarter chord, increase with increasing reduced pitch rate $K$. Higher force coefficients are obtained at fixed $K$ when pivot-axis location moves closer to the leading edge. Also the lift coefficient increases linearly with angle of attack; however, the increase of the induced drag is non-linear. For the pivot-axis location after the three-quarter chord, the force coefficients as a function of angle of attack evolve in opposite trend with increasing $K$, as shown in Fig. 9. Moreover, Fig. 9 shows the force evolution at LE pivot axis for various wing planforms considered in our experiment. The variances in force coefficients are fairly small among these tapered wings. As pivot axis location moves downstream, much smaller variances are predicted.

\section{B. Reduced Pitch Rate and Wing Planform Effects at $K=\mathbf{0 . 3 9}$}

First we consider effect of wing planform shape and reduced pitch rate on force development. The experimental conditions are shown as solid symbols in Fig. 9. Presented here are results for $K=0.39$ in a uniform free-stream flow $U_{\infty}=17.5 \mathrm{~cm} / \mathrm{s}(R e=8.9 \mathrm{k})$.

Figures 10-12 show force evolutions as a function of angle of attack for various wing planforms at $K=0.39$ and leading-edge pivot, mid-chord pivot, and trailing-edge pivot, respectively. The black, blue, and red curves represent rectangular, trapezoidal, and triangular wing, respectively. The solid curves are for cases $K=0.39$ and dotted curves are for cases $K=0$ (denoted by steady). The theoretical results from lifting-line theory for rectangular wings are plotted as green curves.

Figure 10 shows lift and drag coefficients versus angle of attack at leading-edge pivot axis. For lift coefficient at $K=0.39$, non-circulatory apparent mass effects are observed at the beginning and the end of pitch-up phase. During the constant pitch rate phase, lower taper-ratio wing produce slightly higher lift and drag coefficients, beyond the prediction by lifting-line theory and steady flow measurements. The evolutions of lift coefficient for triangular wings do not increase linearly with angle of attack. For the drag coefficient, the pitch rate effects are over-predicted by lifting-line theory at low angle of attack and under-estimated at high angle of attack.

American Institute of Aeronautics and Astronautics 

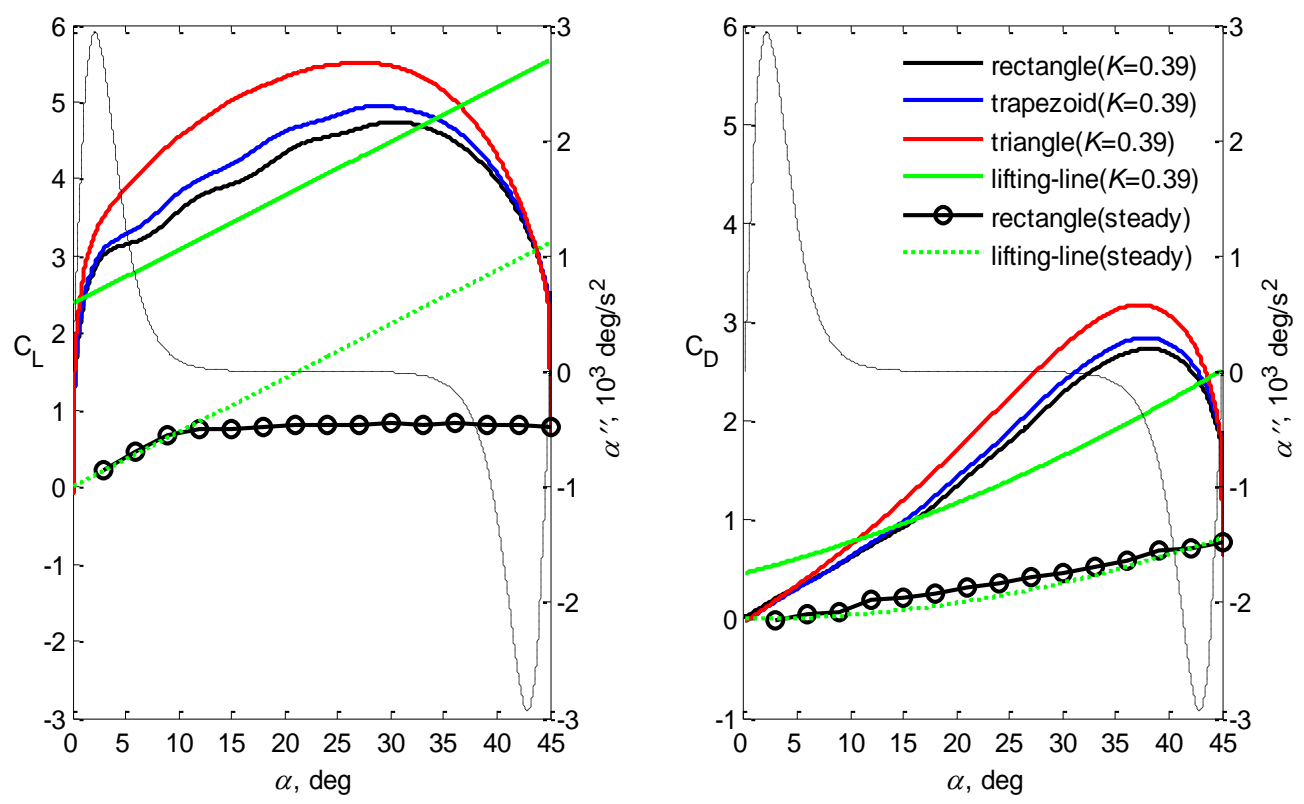

Figure 10. Force evolutions as a function of angle of attack for various wing planforms at $K=0.39$ and leading edge pivot axis: (left) lift coefficient and (right) drag coefficient.

For mid-chord pivot, as shown in Fig. 11, non-circulatory apparent mass effects are absent for three wing planform geometries. The high taper-ratio wing $(\lambda \geq 0.5)$ gives approximately the same lift and drag coefficients, the wing with lower taper-ratio gives higher lift and drag coefficients at high angle of attack, which do not follow the theoretical result. For all wing planforms, measured forces are higher than steady flow measurement by rectangular wing.

For trailing-edge pivot, as shown in Fig. 12, non-circulatory apparent mass effects are found at the beginning and the end of pitching motion. Lift coefficients for taper ratio higher than 0.5 follow the theoretical estimation closely during constant pitch rate phase, whereas drag coefficients are not well predicted. Below 20-degree angle of attack,
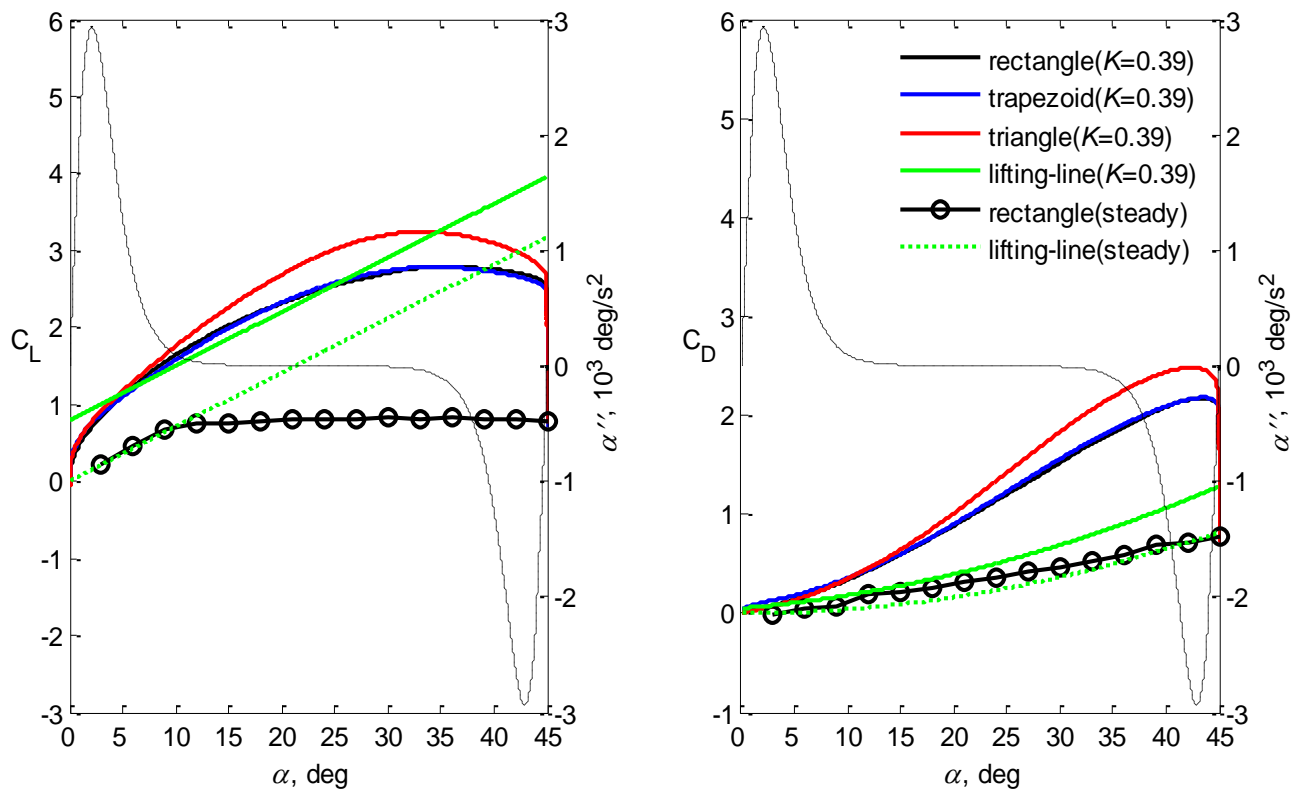

Figure 11. Force evolutions as a function of angle of attack for various wing planforms at $K=0.39$ and mid-chord pivot axis for (left) lift coefficient and (right) drag coefficient

American Institute of Aeronautics and Astronautics 

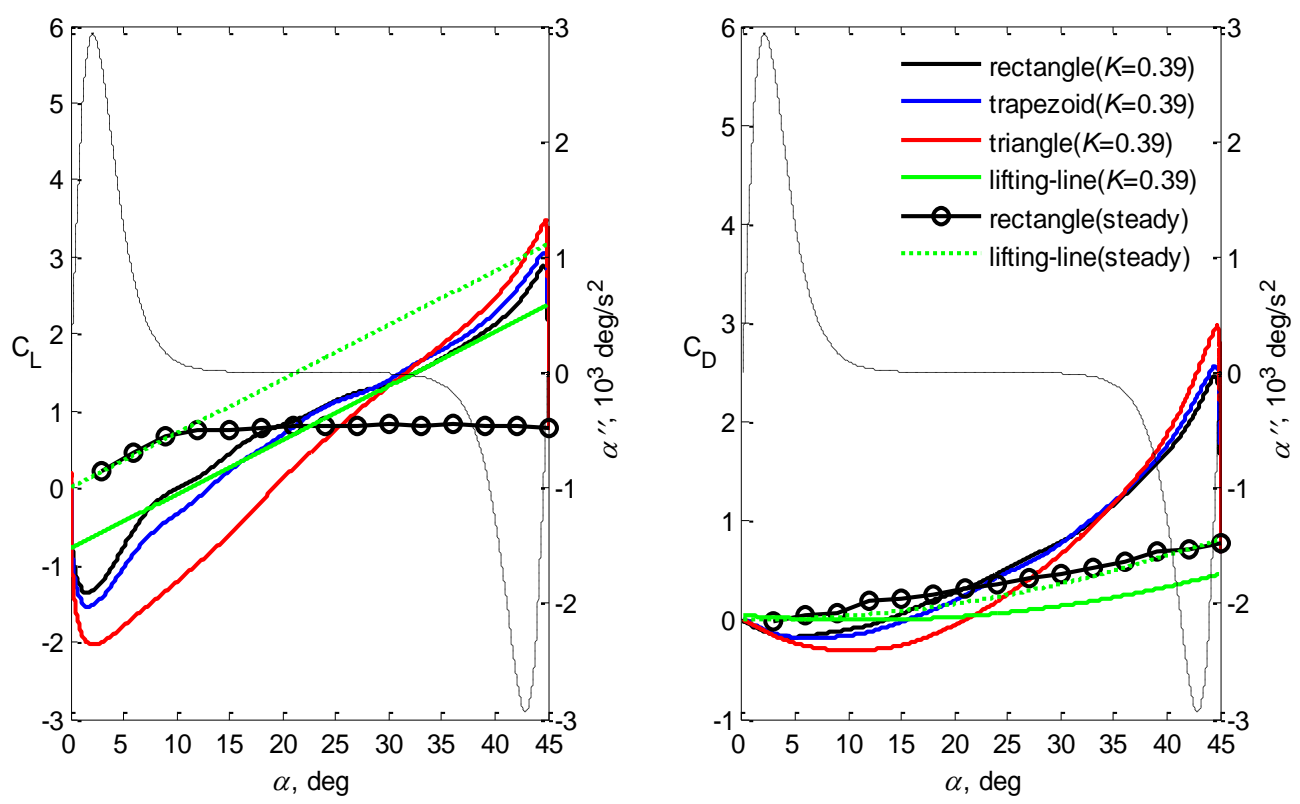

Figure 13 Force evolutions as a function of angle of attack for various wing planforms at $K=0.39$ and trailing edge pivot axis for (left) lift coefficient and (right) drag coefficient

lift and drag coefficients are smaller than the steady flow measurement by rectangular wing; however, force coefficients are larger than the steady flow measurements at higher angle of attack.

Figure 13 shows the pitching moment coefficients about the pivot axis for different wing planforms, the black, blue, and red curves represent rectangular, trapezoidal, and triangular wings, respectively. The steady flow measurement of rectangular wing at mid-chord pivot axis is plotted as circle symbols, and evaluated for both leadingedge and trailing-edge pivot axes. As pivot axis location is at leading edge, negative pitching moments are found in the range of pitch angle, which are consistent with steady flow measurements about corresponding pivot axis. Lower
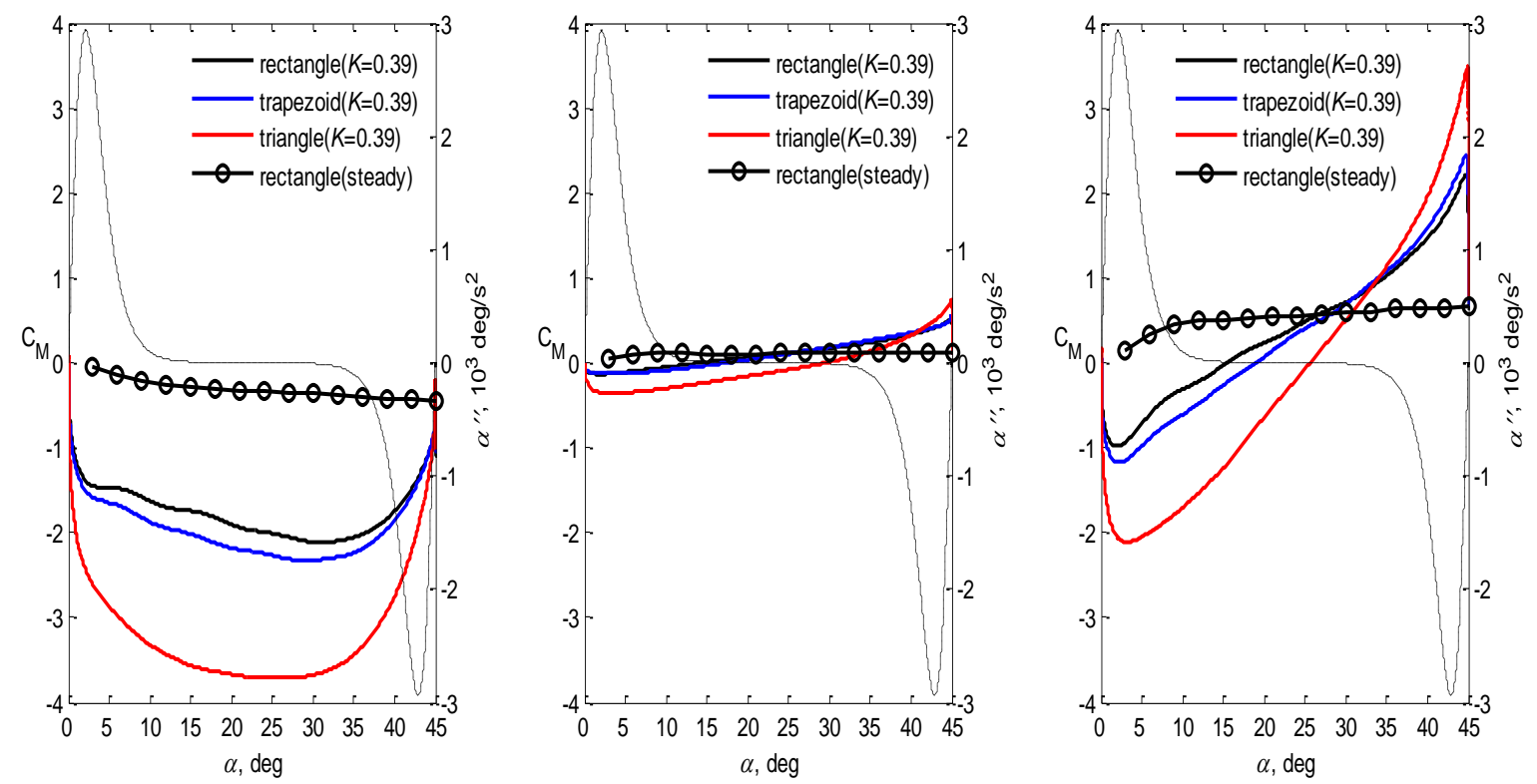

Figure 12 Pitching moment evolution as a function of angle of attack for various wing planforms at $K=$ 0.39 and (left) leading edge, (middle) mid-chord, and (right) trailing edge.

American Institute of Aeronautics and Astronautics 

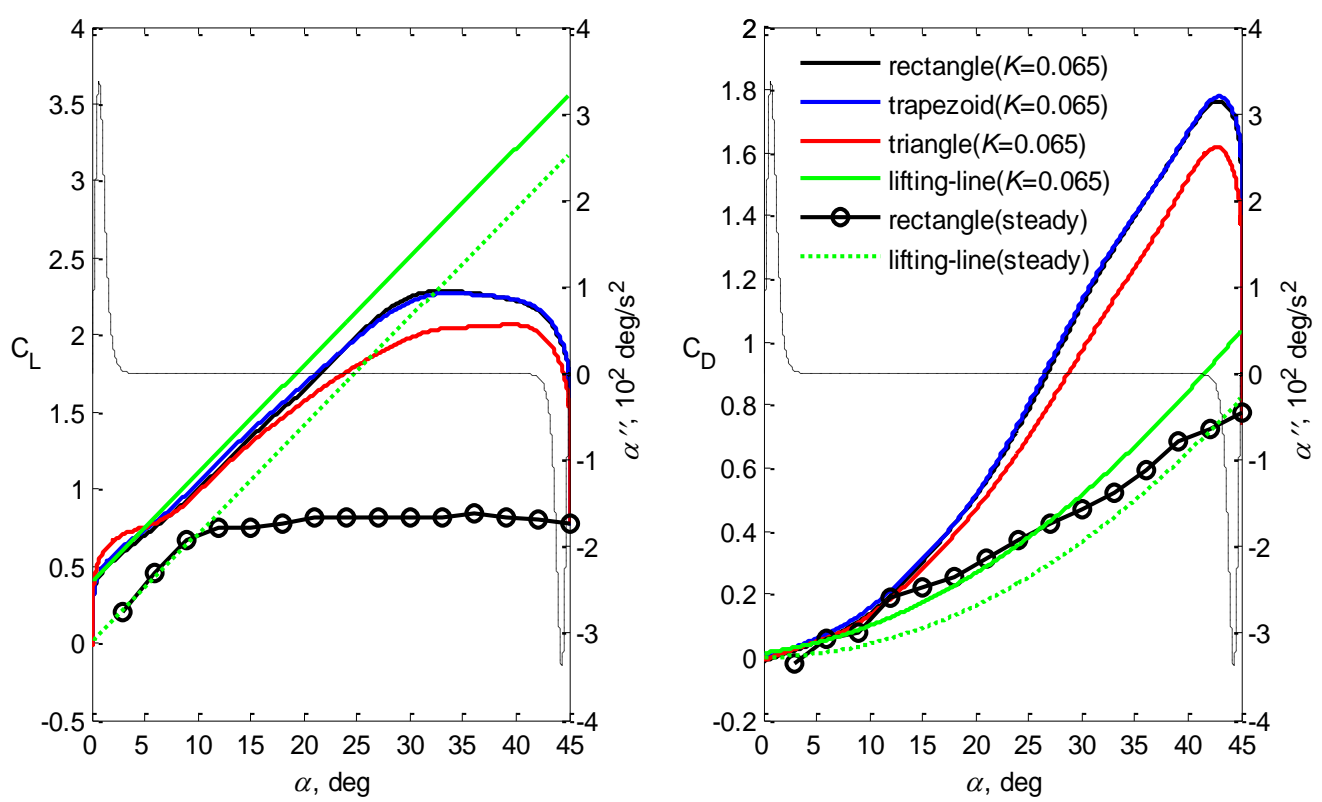

Figure 14. Force evolutions as a function of angle of attack for different wing planforms at $K=0.065$ and leading edge pivot axis: (left) lift coefficient and (right) drag coefficient.

taper ratio gives higher magnitude of pitching moment coefficient. For mid-chord pivot, high taper ratio wings $(\lambda \geq$ $0.5)$ give the similar pitching moment coefficients; the wing with lower taper ratio gives higher magnitude of pitching moment coefficients but all magnitudes are less than one. Moreover, negative pitching moment coefficient is observed at low angle of attack, which is contrary to the positive pitching moment coefficient in steady flow. For trailing-edge pivot axis, negative pitching moment coefficients are also observed at low angle of attack with much larger amplitude than that at mid-chord pivot axis.
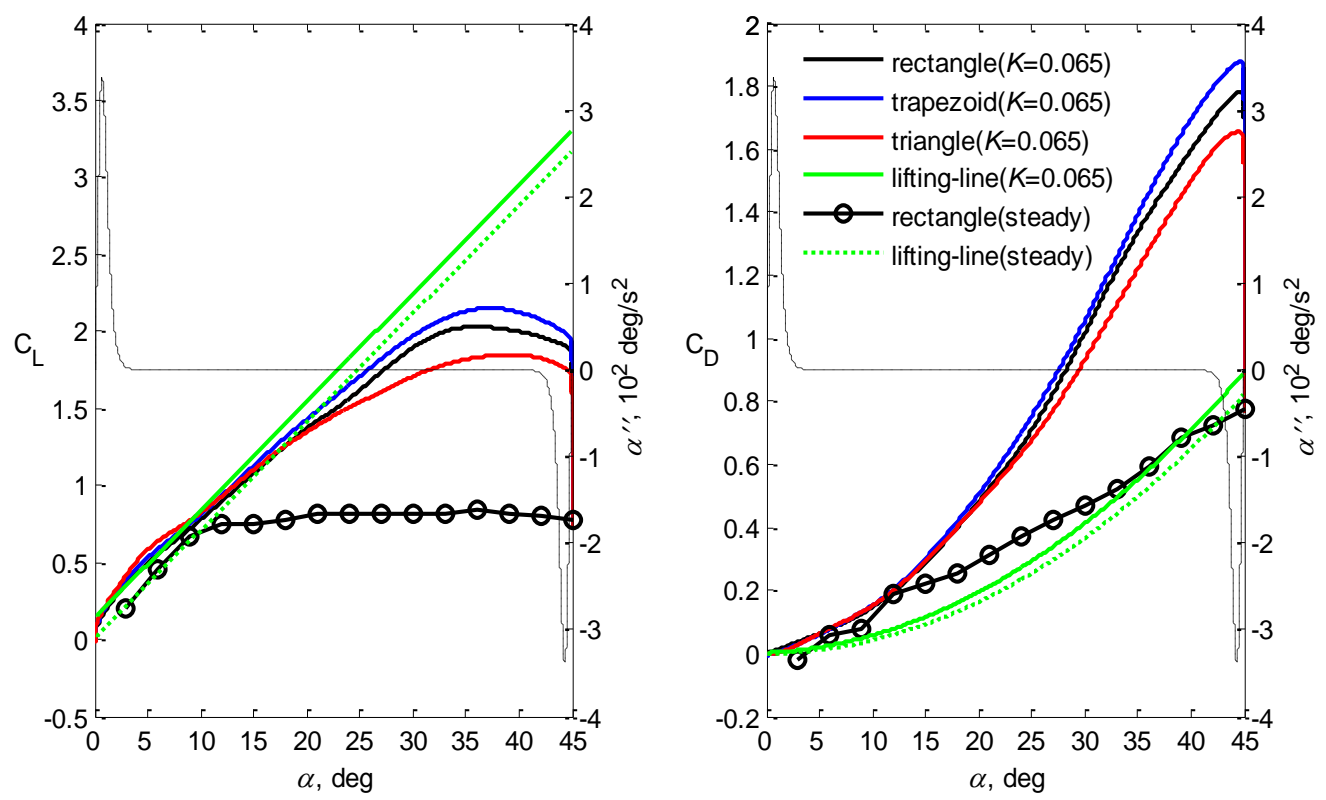

Figure 15. Force evolutions as a function of angle of attack for different wing planforms at $K=0.065$ and mid-chord pivot axis: (left) lift coefficient and (right) drag coefficient.

American Institute of Aeronautics and Astronautics 

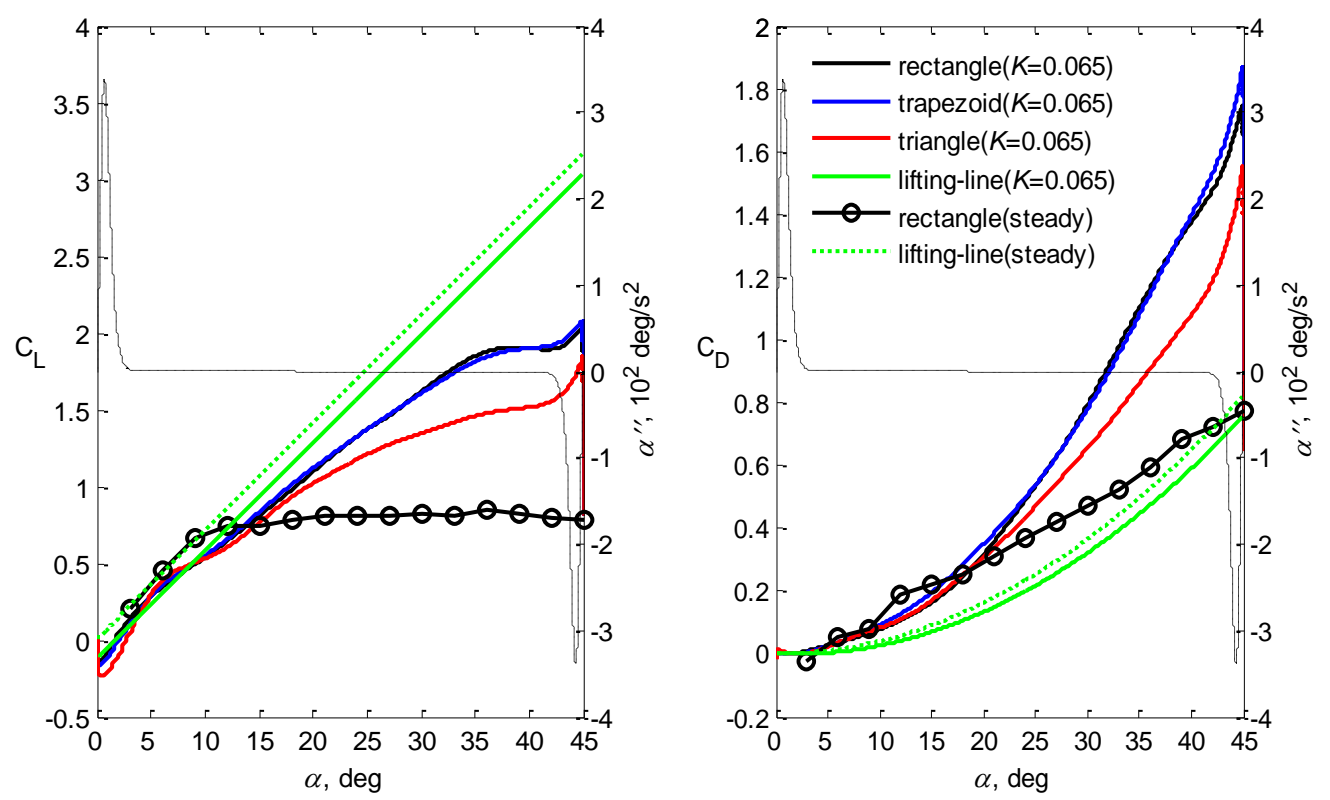

Figure 16. Force evolutions as a function of angle of attack for different wing planforms at $K=0.065$ and trailing edge pivot axis: (left) lift coefficient and (right) drag coefficient.

\section{Reduced Pitch Rate and Wing Planform Effects at $K=\mathbf{0 . 0 6 5}$}

Figures 14-16 show the force coefficients as a function of angle of attack for various wing planforms and pivot location at low reduced pitch rate $K=0.065$. The similar legends as $K=0.39$ are used in these figures. The black, blue, and red curves represent rectangular, trapezoidal, and triangular wing, respectively. The solid curves are for
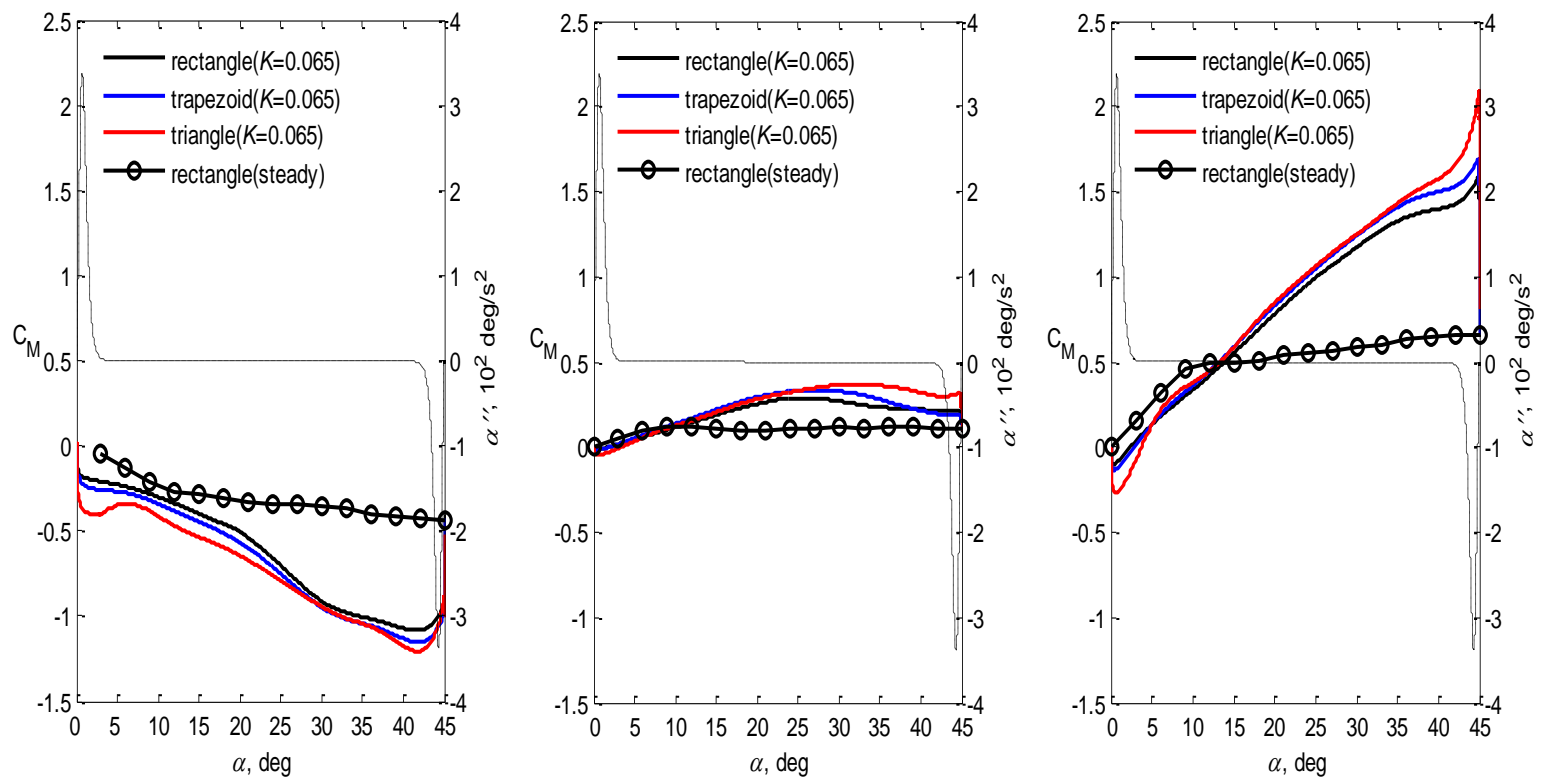

Figure 17. Pitching moment coefficient about the pivot location as a function of angle of attack for different wing planforms at $K=0.065$ and (left) leading edge pivot, (middle) mid-chord pivot, and (right) trailing edge pivot.

American Institute of Aeronautics and Astronautics 


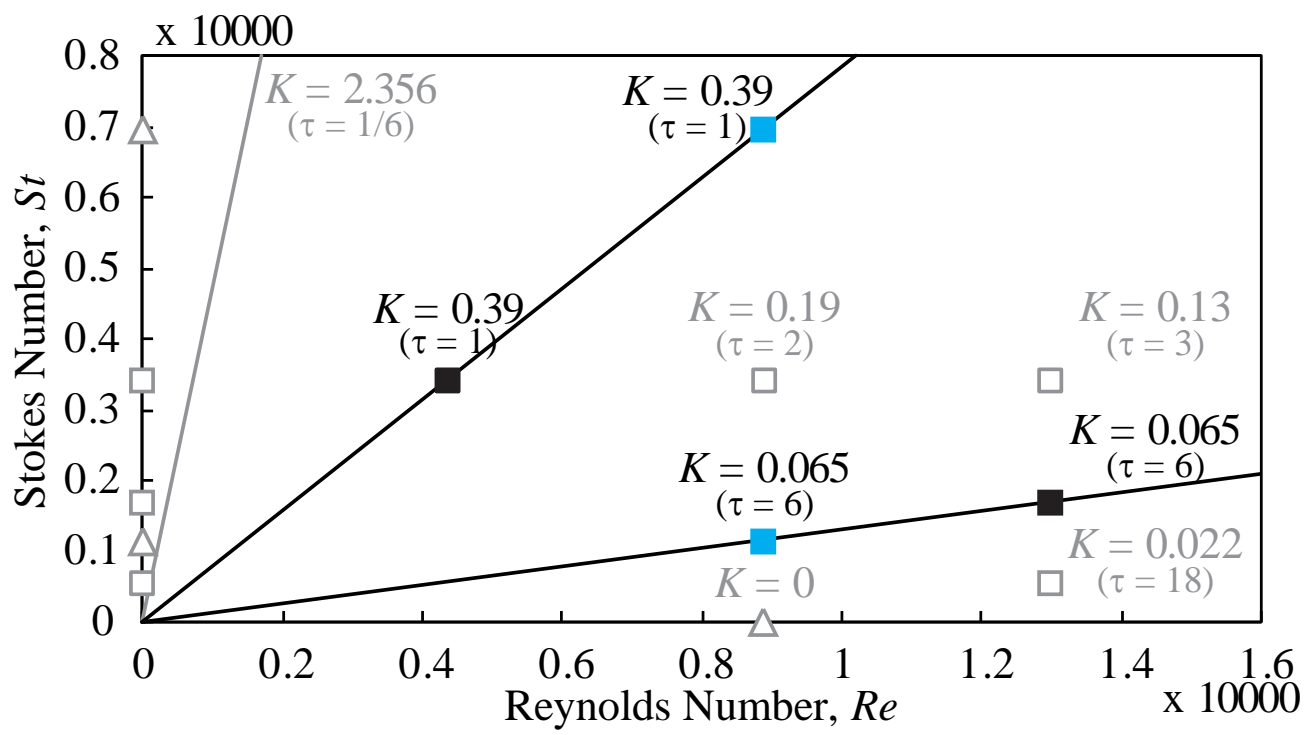

Figure 18. Experimental conditions for the study of Reynolds number and smoothing transient effect

cases $K=0.065$ and dotted curves are for cases $K=0$ (denoted by "steady"). The theoretical results from lifting-line theory for rectangular wings are plotted as green curves.

In contrast to the high reduced-pitch-rate cases $K=0.39$, the rectangular and trapezoidal wings, which taper ratio is higher than 0.5, produce very similar force dependence with angle of attack at both LE and TE pivot-axis location. In addition, the non-circulatory effects at beginning and the end of the pitching motion for the triangular wing are stronger than that for the other wing planforms, which is similar to the higher $K=0.39$ case. However, during the constant pitch rate, the triangular wing produces less force coefficients at high angle of attack.

\section{Reynolds Number and Smoothing kinematics Effects}
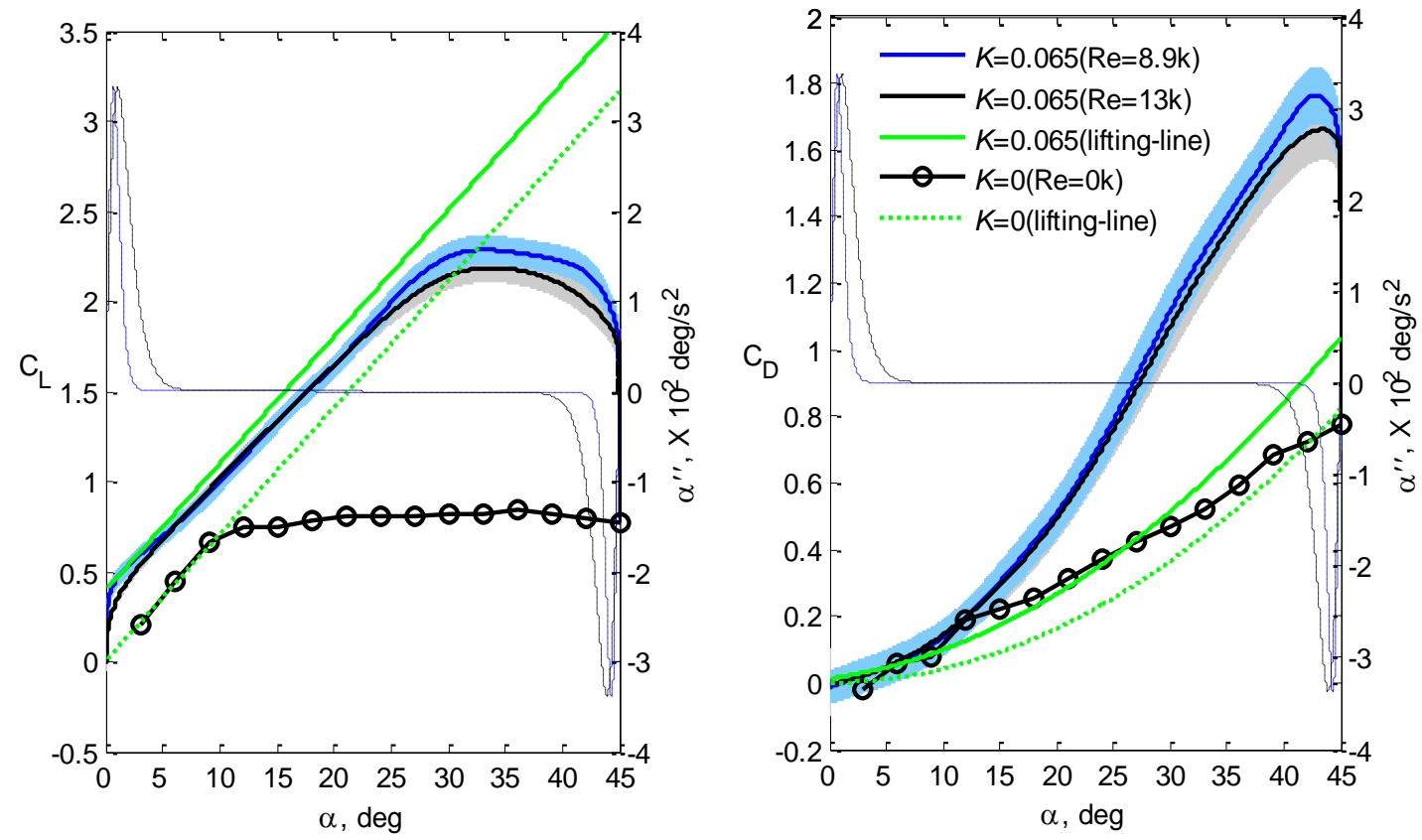

Figure 19. Force evolution as a function of angle of attack for rectangular wing and $K=0.065$ at leading edge pivot (left) lift coefficient (right) drag coefficient.

American Institute of Aeronautics and Astronautics 

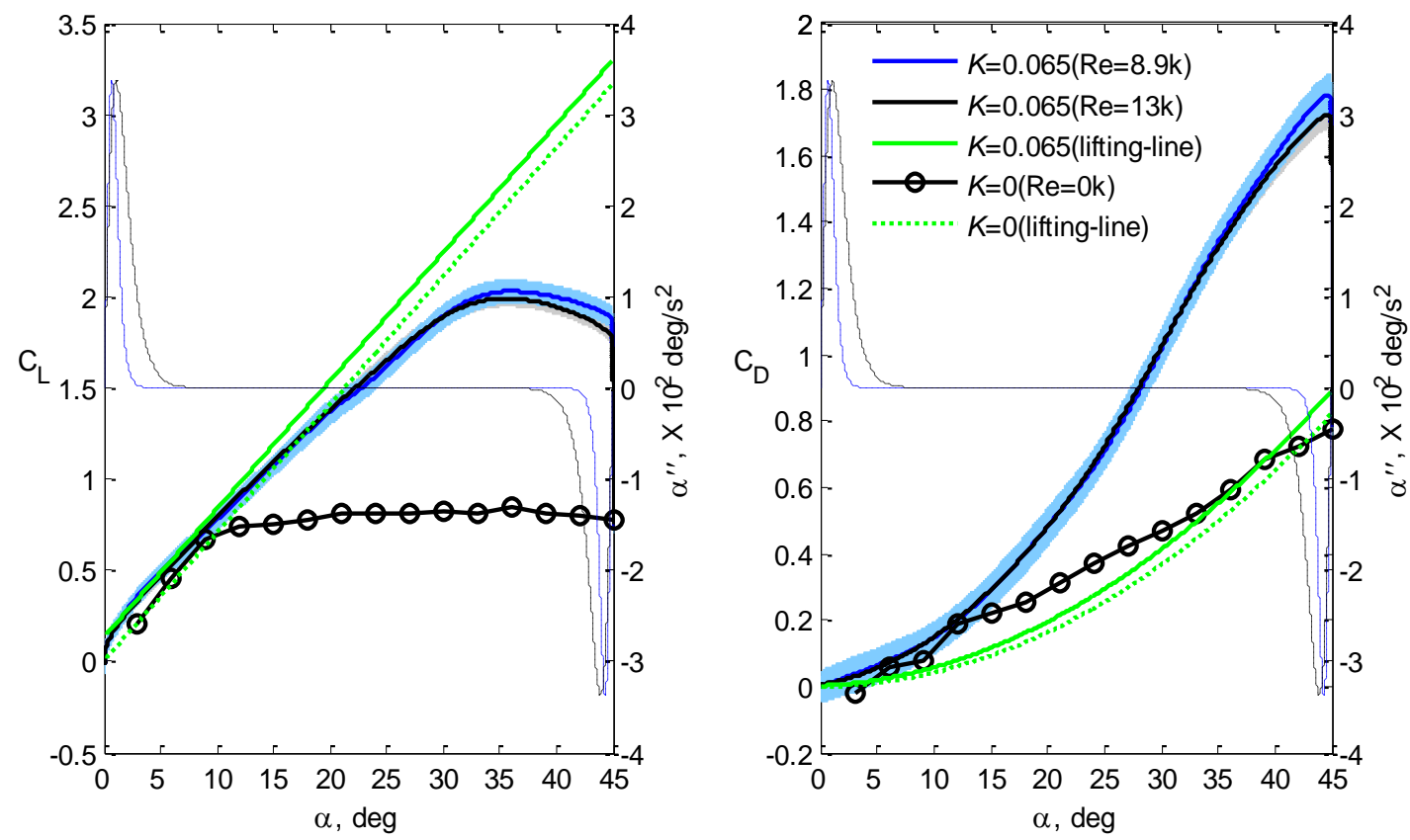

Figure 21. Force evolution as a function of angle of attack for rectangular wing and $K=0.065$ at midchord pivot (left) lift coefficient (right) drag coefficient.

The investigation of Reynolds number effect was conducted using different wing kinematics and free-stream velocity with the same reduced pitch rate. There are two reduced pitch rates considered here for rectangular wings at various pivot axes, which are $K=0.065$ and 0.39 . The parameter values are shown in Fig. 18 as solid symbols. For $K$ $=0.065$, the comparison was made between the combination of $R e=13 \mathrm{k}\left(U_{\infty}=25.6 \mathrm{~cm} / \mathrm{s}\right)$ and $S t=1.7 \mathrm{k}\left(\alpha_{m}^{\prime}=37.5\right.$
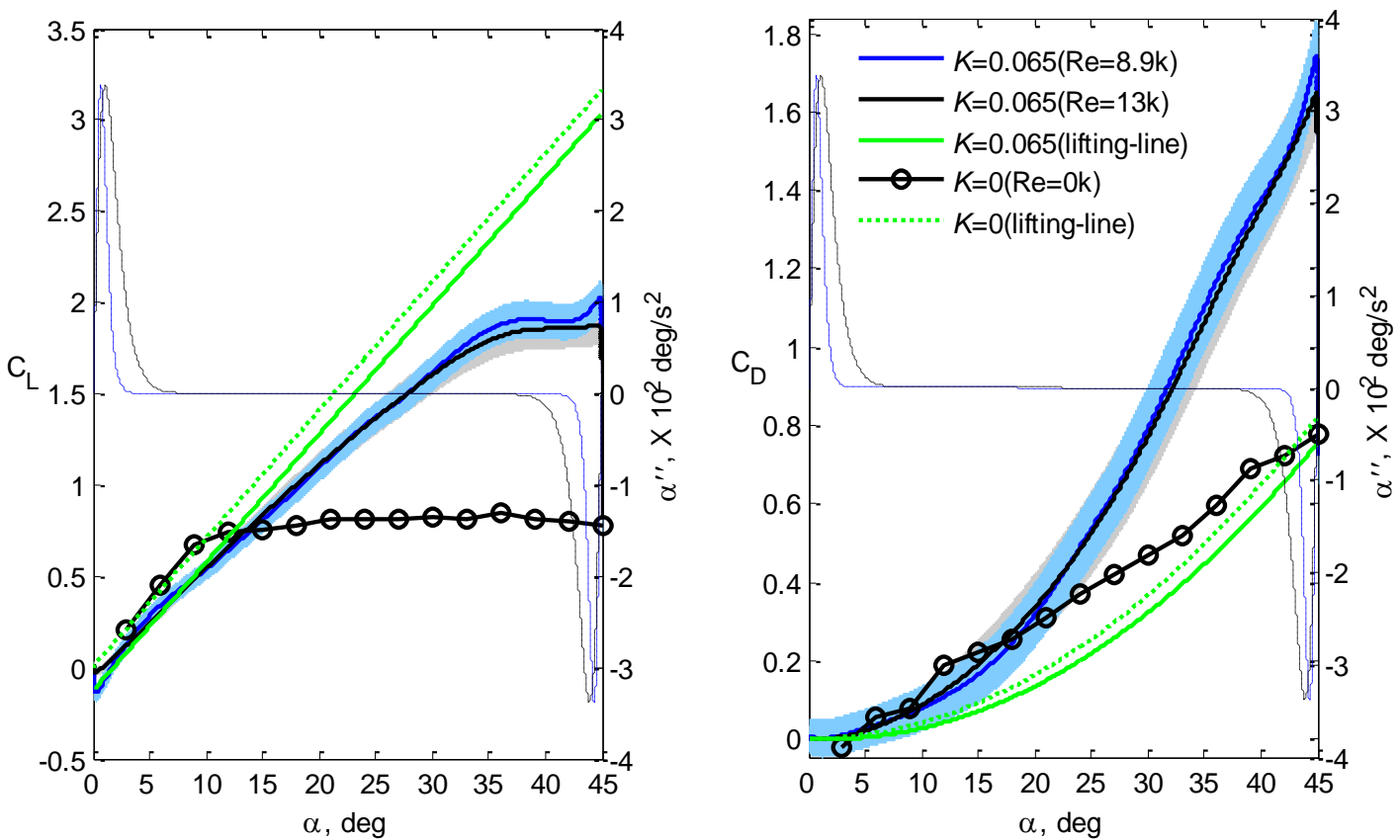

Figure 20. Force evolution as a function of angle of attack for rectangular wing and $K=0.065$ at trailing edge pivot (left) lift coefficient (right) drag coefficient.

American Institute of Aeronautics and Astronautics 

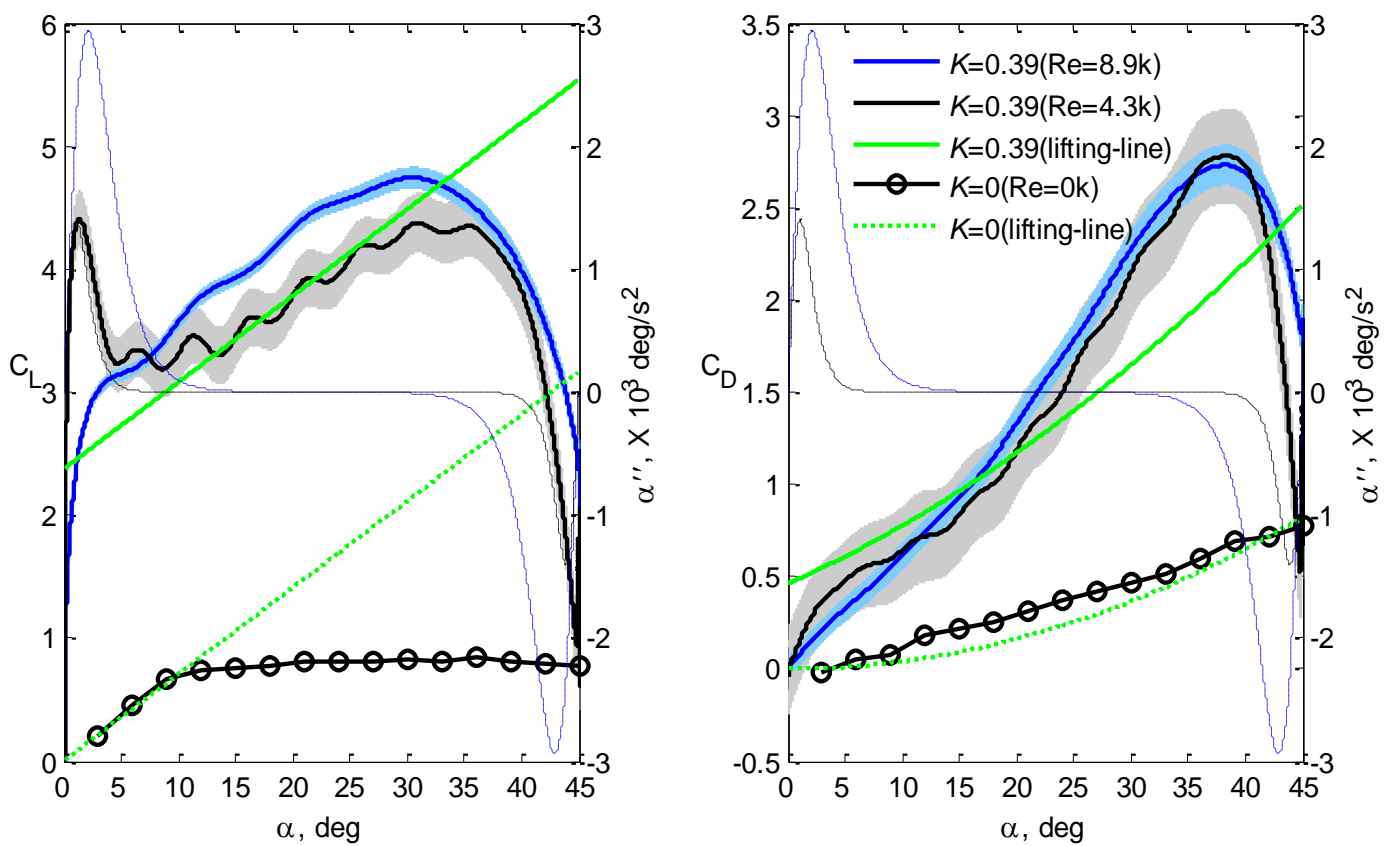

Figure 23. Force evolution as a function of angle of attack for rectangular wing and $K=0.39$ at leading edge pivot (left) lift coefficient (right) drag coefficient.

$\% / \mathrm{s})$ and the combination of $R e=8.9 \mathrm{k}\left(U_{\infty}=17.5 \mathrm{~cm} / \mathrm{s}\right)$ and $S t=1.1 \mathrm{k}\left(\alpha_{m}^{\prime}=25.6 \%\right)$. For $K=0.39$, the comparison was made between the combination of $R e=8.9 \mathrm{k}\left(U_{\infty}=17.5 \mathrm{~cm} / \mathrm{s}\right)$ and $S t=7.0 \mathrm{k}\left(\alpha_{m}^{\prime}=155 \% \mathrm{~s}\right)$ and the combination of $R e=4.3 \mathrm{k}\left(U_{\infty}=8.6 \mathrm{~cm} / \mathrm{s}\right)$ and $S t=3.4 \mathrm{k}\left(\alpha_{m}^{\prime}=76.5 \%\right)$. All kinematics have different smoothing maneuvering at
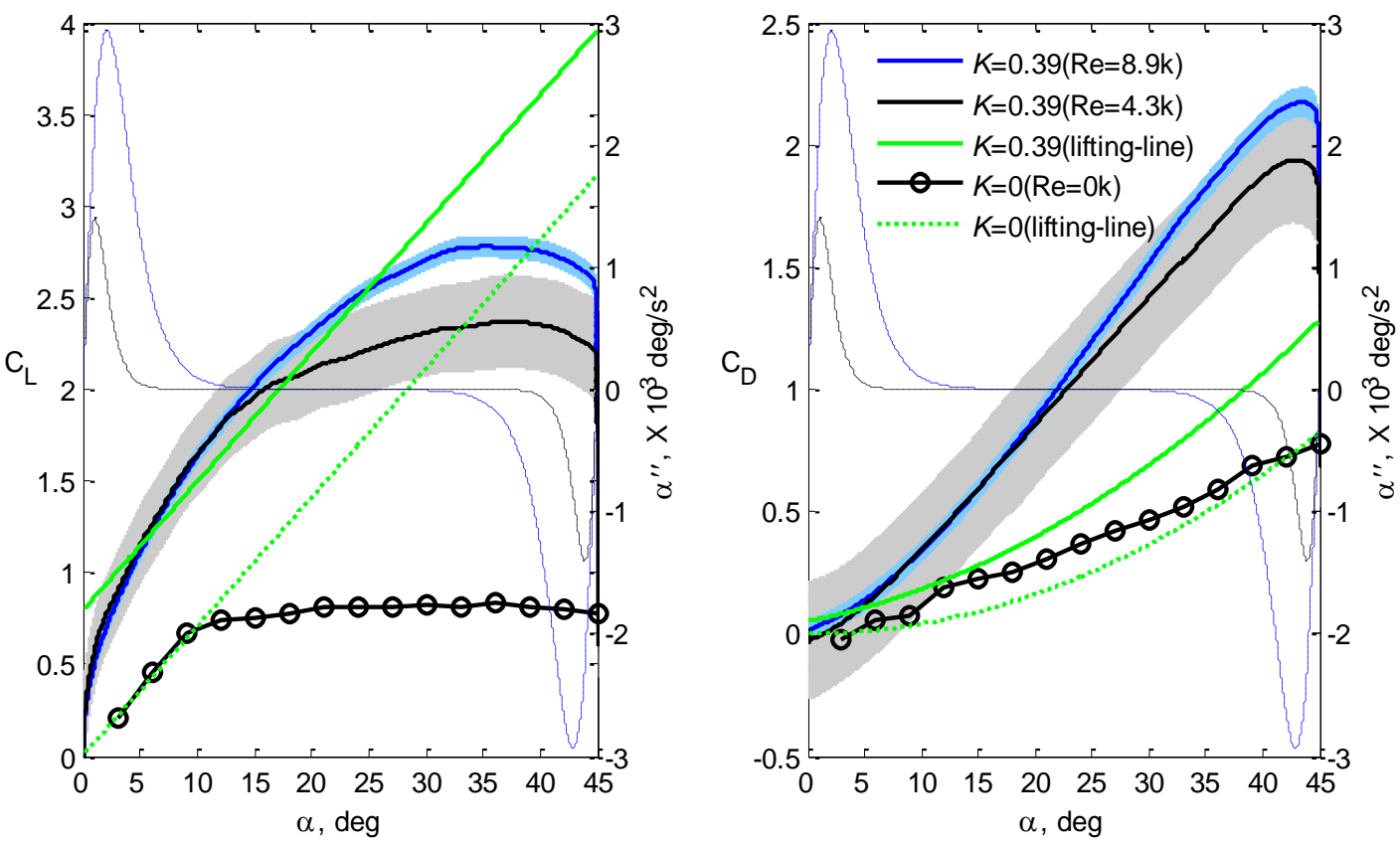

Figure 22. Force evolution as a function of angle of attack for rectangular wing and $K=0.39$ at mid-chord pivot (left) lift coefficient (right) drag coefficient.

American Institute of Aeronautics and Astronautics 

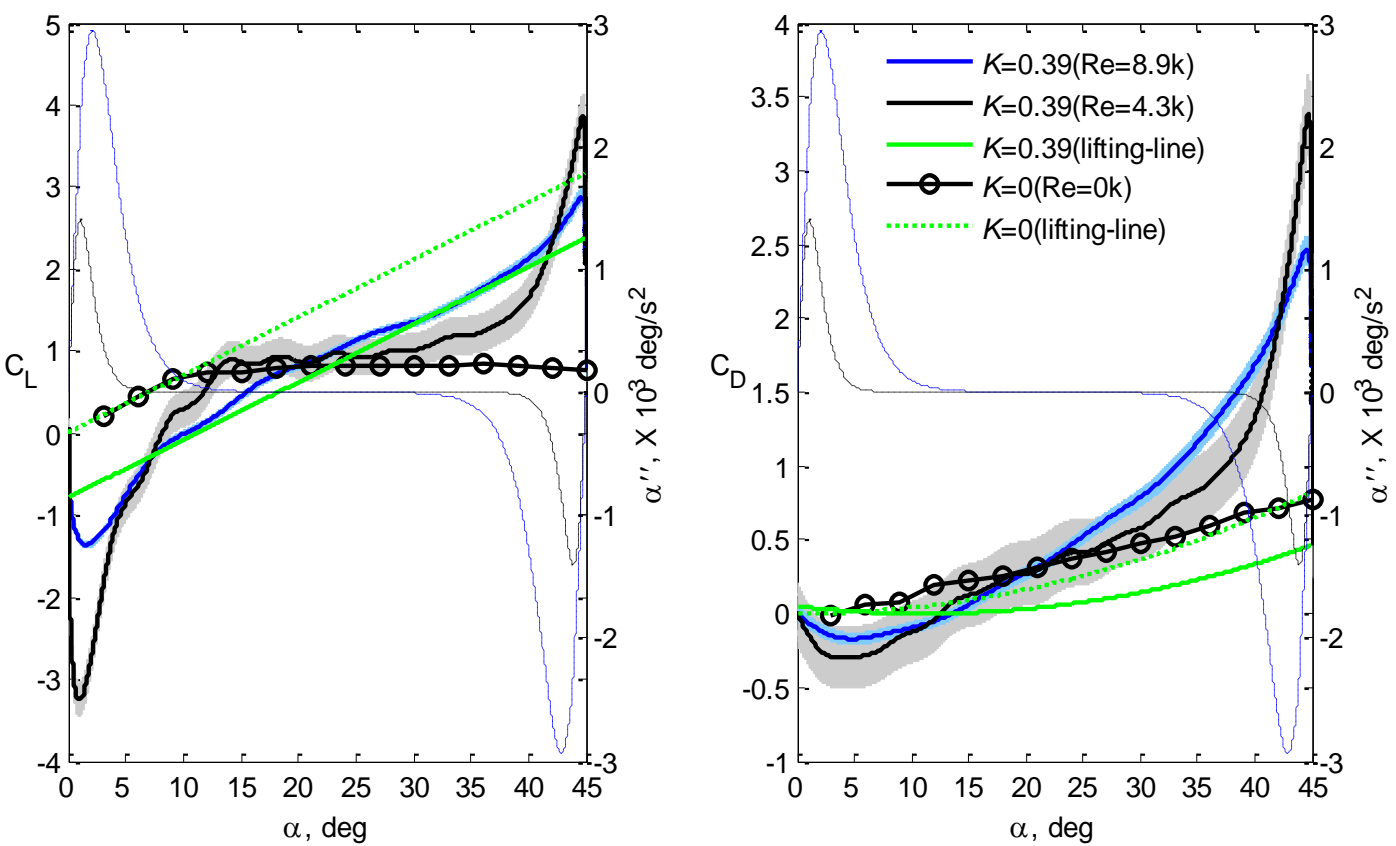

Figure 24. Force evolution as a function of angle of attack for rectangular wing and $K=0.39$ at trailing pivot (left) lift coefficient (right) drag coefficient.

beginning and the end of pitching. The shaded area presents the standard deviation in the measurement. The pitching accelerations are plotted as thin dashed curves.

For $K=0.065$, as shown in Figs. 19-21, all pivot-axis location show similarity of lift and drag coefficients with respect to angle of attack at given constant reduced pitch rate. Close examination of two data reveals several distinct features. Firstly, non-circulatory apparent-mass effect is evident for both leading-edge and trailing-edge pivots in $R e$ $=8.9 \mathrm{k}$, caused by pitching acceleration, and is not observed in $R e=13 \mathrm{k}$ as documented by Yu and Bernal (2013). The discrepancy is due to pitching acceleration duration. Both kinematics has the same maximum acceleration. but pitching acceleration duration $\left(2 t_{a}\right)$ for $R e=8.9 \mathrm{k}$ is equal to half of one convective time, which is two times less than pitching acceleration duration for $R e=13 \mathrm{k}$. Secondly, despite the variation of pivot-axis location, the occurrence of noncirculatory effect at the beginning of the motion has little impact on rotation rate effect before the saturation of forces during constant pitch-rate phase. The force curves are similar up to 20-degree angle of attack for leading edge pivot, 30-degree angle of attack for other two pivot axes.

Unlike low reduced pitch rate $K=0.065$, force curves in Figs. 22-24 for $K=0.39$ show much pronounced variations as pivot-axis location at both leading edge and trailing edge. For mid-chord pivot, the force curves are still in a good agreement below 12-degree angle of attack. The variation at lower angle of attack is caused by non-circulatory effect being associated with pitching acceleration; the associating vortical structure is the starting vortex. Recall the conditions used to generate wing kinematics from the Table, the kinematics in $R e=8.9 \mathrm{k}$ has both maximum acceleration and acceleration duration being twice as large as that in $R e=4.3 \mathrm{k}$. As a result, the strength of starting vortex in $R e=8.9 \mathrm{k}$ would enhance the rotation rate effect during constant pitch-rate phase and shift the lift-coefficient curve upward, but has very little impact on drag-coefficient curve. For trailing edge pivot, the slope of lift-coefficient curve in $R e=8.9 \mathrm{k}$ is in reasonable agreement with theoretical estimation. In addition, both kinematics have pitching acceleration durations less than half of one convective time.

\section{Concluding Remarks}

In this paper we report new experimental measurements of aerodynamic forces on flat plate wings undergoing a pitch ramp motion. Lift measurement results are in reasonable agreement with steady linear potential flow theory. Measured drag coefficients are significantly larger than the prediction by the theory. The theoretical analysis includes rotation rate effects and finite aspect ratio effects. Features like the effect of pivot location, wing planform geometry are well captured at low reduced pitch rate. At high pitch rate the agreement is not as good and enhancements of the

American Institute of Aeronautics and Astronautics 
theory are being developed to account for these effects. Effects of non-circulatory apparent mass and rotation rate are independent at onset of pitching motion and low angle of attack, indicating the application of superposition principle on forces.

\section{References}

${ }^{1}$ Ol, M. V., Altman, A., Eldredge, J. D., Garmann, D. J., Lian, Y., Résumé of the AIAA FDTC Low Reynolds Number Discussion Group's Canonical Cases, 48th AIAA Aerospace Sciences Meeting, Orlando, FL, AIAA 2010-1085.

${ }^{2}$ Granlund, K., Ol, M., Garmann, D., Visbal, M., Bernal, L., "Experiments and Computations on Abstractions of Perching", 28th AIAA Applied Aerodynamics Conference, Chicago, IL, AIAA 2010-4943.

${ }^{3}$ Baik, Y. S., Aono, A. Rausch, J. M., Bernal, L. P., Shyy, W., "Experimental Study of a Rapidly Pitched Flat Plate at Low Reynolds Number", ”, 40th AIAA Fluid Dynamics Conference, AIAA 2010-4462.

${ }^{4}$ Ol, M. V., Eldredge, J. D., Wang, C., "High-Amplitude Pitch of a Flat Plate: an Abstraction of Perching and Flapping", International Journal of Micro Air Vehicles, Vol. 1, No. 3, Sep. 2009.

${ }^{5}$ Eldredge, J. D. and Wang, C., “ A Computational Study of a Canonical Pitch-up, Pitch-down Wing Maneuver”, 39th AIAA Fluid Dynamics Conference, AIAA 2009-3687.

${ }^{6}$ Garmann, D. J. and Visbal, M. R., "High-Fidelity Simulations of Transitional Flow Over Pitching Airfoils", 39th AIAA Fluid Dynamics Conference, AIAA 2009-3693.

${ }^{7}$ Lian, Y. and OL, M. V., "Computation and Experiments on a Low Aspect Ratio Pitching Flat Plate", 48th AIAA Aerospace Sciences Meeting Including the New Horizons Forum and Aerospace Exposition, AIAA paper 2010-0385.

${ }^{8}$ Harper, P.W. and Flanigan, R.E., "The Effect of Change of Angle of Attack on the maximum Lift of a small Model", NACA TN 2061, March 1950.

${ }^{9}$ McCroskey, W.J. and Philippe, J.J., "Unsteady Viscous Flow on oscillating Airfoils", AIAA Journal, Vol. 13, No. 1, Jan. 1975.

${ }^{10}$ Currier, J.M. and Fung, K.Y., “Analysis of the Onset of Dynamic Stall”, AIAA Journal, Vol. 30, No. 10, October 1992.

${ }^{11}$ Ellington, C.P., Berg, C.V.D., Willmott, A.P., and Thomas, A.L.R., "Leading Edge Vortices in Insect Flight", Nature (London), Vol. 384, 1996, pp. 626-630.

${ }^{12}$ Birch, J.M. and Dickinson, M.H., "Spanwise Flow and the Attachment of the Leading Edge Vortex on Insect Wings", Nature (London), Vol. 412, 2001, pp. 729-733.

${ }^{13}$ Shyy, W. and Liu, H., "Flapping Wings and Aerodynamic Lift: The Role of Leading Edge Vortices", AIAA Journal, Vol. 45, No. 12, Dec. 2007.

${ }^{14}$ Glauert, H. "The Elements of Aerofoil and Airscrew Theory”, Cambridge University Press, 1947.

${ }^{15}$ Leishman, J.G., Principle of Helicopter Aerodynamics, $2^{\text {nd }}$ ed., Cambridge University Press, New York, 2006.

American Institute of Aeronautics and Astronautics 